\title{
Excitation Modes for Fourier Transform-Ion Cyclotron Resonance Mass Spectrometry
}

\author{
Lutz Schweikhard \\ Institute of Physics, University of Mainz, Mainz, Germany
}

\author{
Alan G. Marshall \\ Department of Chemistry and Biochemistry, The Ohio State University, Columbus, Ohio, USA
}

\begin{abstract}
Various geometric configurations for the excitation of coherent ion motion in Fourier transform-ion cyclotron resonance mass spectrometry (FT-ICR/MS) are analyzed (in some cases for the first time) with unified notation. The instantaneous power absorption, $\mathbf{F} \cdot \mathbf{v}$, in which $\mathbf{v}$ is ion velocity and $\mathbf{F}$ the force produced by the applied excitation electric field (harmonic, single frequency, on-resonance, in-phase), is time averaged and then set equal to the time rate of change of ion total (cyclotron + magnetron + trapping) energy, to yield a differential equation that is readily solved for the (time-dependent) amplitude of each of the various ion motions. The standard FT-ICR excitation (namely, radial dipolar) is reviewed. The effects of quadrature and radial quadrupolar excitation on ion radial (cyclotron and magnetron) motions are also reviewed. Frictional damping is shown to decrease the ion cyclotron orbital radius and trapping amplitude but increase the magnetron radius. Feedback excitation (i.e., excitation at the simultaneously detected ion cyclotron orbital frequency of the same ion packet) is introduced and analyzed as a means for exciting ions whose cyclotron frequency changes during excitation (as for relativistically shifted low-mass ions). In contrast to conventional radial dipolar excitation, axial dipolar excitation of the trapping motion leads to a mass-dependent ion motional amplitude. Parametric (i.e., axial quadrupolar) excitation is shown to produce an exponential increase in the ion motional amplitudes (hyperbolic sine and hyperbolic cosine amplitude for cyclotron and magnetron radii, respectively). More detailed consideration of parametric excitation leads to an optimal ion initial radial position in parametric-mode FT-ICR/MS. ( $/$ Am Soc Mass Spectrom 1993, 4, 433-452)
\end{abstract}

$\mathrm{T}$ echnique developments in Fourier transform-ion cyclotron resonance mass spectrometry (FTICR/MS) have recently been reviewed $[1,2]$; in addition, more than a dozen reviews of FT-ICR/MS analytical and chemical applications are also cited therein. FT-ICR experiments are conveniently classified according the ion trap wiring configuration and operating mode, which in turn determine which of the various "natural" cyclotron, magnetron, and trapping frequencies (or combinations of those frequencies) of ion motion are observed. Unfortunately, prior theoretical descriptions (where available) of these operating modes are distributed over several reports by different workers using different notation and formalism. For example, radial dipolar ion excitation for FT-ICR/MS in the conventional six-electrode orthorhombic geometry has previously been analyzed in detail $[3,4]$. However, formal treatment of other excitation geometries for use with FT-ICR/MS is still lacking.

In this study, we provide a unified description, with

Address reprint requests to Alan G. Marshall, The Ohio State University, 120 West 18 th Avenue, Columbus, OH 43210. consistent notation, of prior (and some new) excitation modes in the quadrupolar electrostatic trapping potential approximation (appropriately scaled for a cubic ICR ion trap) and spatially linear (dipolar, i.e., uniform electric field) or quadrupolar excitation potential. Our approach is simply to equate the instantaneous singlefrequency power absorption by an ion (averaged over one cycle of its oscillation) to the time derivative of the (known) ion energy at the ion position in a quadrupolar trapping potential field. We are then able to compute the motional amplitude of an ion subjected to any of several excitation configurations as a function of time during the excitation period; the detected post-excitation signal from such ions is then directly related to the amplitude of the appropriate ion motion at the end of the excitation period. We begin by reviewing the conventional excitation geometry (including both dipolar and quadrature excitation). We then analyze ion relaxation modeled by a frictional damping force and its conceptual "inverse," "feedback" excitation (see below). We then proceed to treat dipolar excitation of the trapping (axial) ion motion as well as so-called parametric excitation of both axial and radial motions. 
a
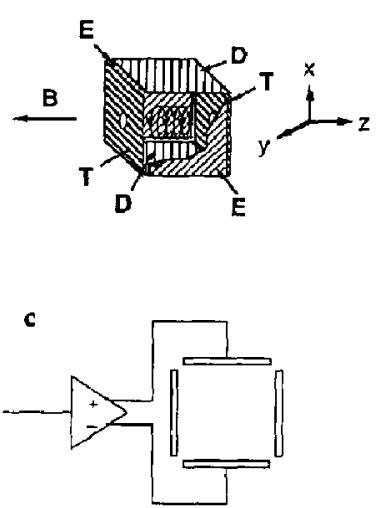

b

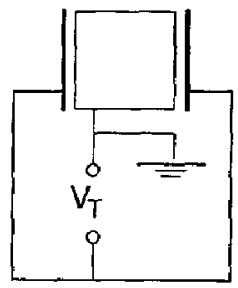

d

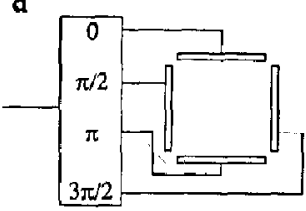

f

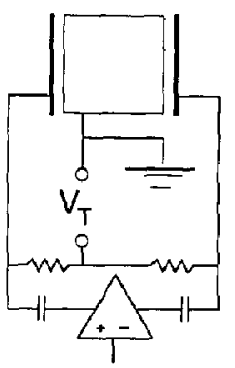

h

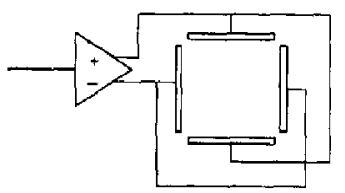

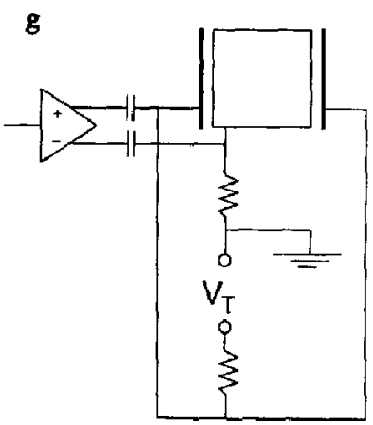

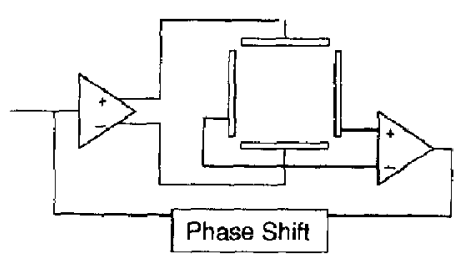

Figure 1. Electrical connections for a cubic ICR ion trap for different excitation modes: (a) three-dimensional view of cubic trap; (b) electrostatic DC trapping potential (trapping electrodes on left and right, side electrodes in front and back); (c) standard (radial dipolar) excitation (cross section thruugh side electrodes in $x y$ plane perpendicular to applied magnetic field); (d) quadrature excitation (cross section as in c); (e) feedback excitation (cross section as in c): left and right electrodes are for detection; top and bottom electrodes are for excitation; (f) axial dipole excitation (as in b); (g) parametric excitation (as in b); (h) azimuthal quadrupolar excitation (cross section as in c). (See text for explanation of symbols.)

Finally, we consider radial $(x y)$ quadrupolar excitation, which allows coupling of the cyclotron and magnetron motions.

To describe the various excitation geometries (Figure 1), we must first consider the various possible trapping geometries. Figure la shows a three-dimensional view of a cubic trap [5], for which trapping is achieved by applying a static electric potential to each of the "trapping" or "end cap" electrodes (T in Figure 1a), as shown in Figure $1 \mathrm{~b}$. The wiring configurations, Figure $1 b-h$, for other trapping geometries (e.g., orthorhombic or tetragonal $[3,4,6]$, cylindrical $[7,8]$, or hyperbolic with segmentation of ring electrode [9-14] are conceptually the same, except that the electrode surfaces may be curved and/or rectangular rather than square.

Excitation modes may be classified as follows. In the standand excitation mode (radial dipolar excitation) (Fỉgure 1c), excitation voltages of opposite phase are applied to two opposed "side" electrodes. This excitation geometry was used in the very first FT-ICR experiment [15] and remains the most popular. It provides for excitation of the cyclotron motion with minimal interference from other ion motional modes and leaves the other pair of upposed side electrodes available for pickup of the induced ion signal.
In the quadrature excitation geometry [16], excitation voltages are applied to all four side electrodes, but with phase differing by $\pi / 2$ between any two adjoining electrodes, as shown in Figure 1d. In this way, a circularly polarized excitation electric field is created, with two advantages: (1) excitation can be made selective for positive or negative ions because ions of one or the other charge sign undergo cyclotron orbital motion, with one or the other rotation sense; and (2) the excitation voltage amplitude need only be half that for linearly polarized excitation (e.g., standard radial dipolar excitation) because linearly polarized radiation can be analyzed as the sum of two counterrotating components, only one of which rotates in the same sense as the ions of the charge sign of interest, so that half of the linearly polarized excitation amplitude is unavailable for excitation [16, 17]. All four side plates of a cubic trap are engaged in quadrature excitation, as is also the case in parametric and azimuthal quadrupolar excitation (see below). Ion detection may be accomplished in three ways: (1) segmentation of the side electrodes to provide four electrodes for excitation and additional electrodes for detection; (2) time-sharing between excitation and detection on the same electrode configuration (i.e., switching the connections from excitation amplifier to detection preamplifier at the end 
of the excitation period); or (3) axial transfer of ions from the excitation trap to another trap for detection.

We discuss frictional deexcitation as a model for the relaxation of coherent ion motion due to collisions. We then propose a new feedback excitation (Figure 1e), the mathematical time inverse of frictional damping. Feedback excitation tracks the ICR frequency of an ion so as to keep the excitation frequency "locked" to the ICR orbital frequency during excitation. Feedback excitation could be useful when the ICR orbital frequency changes during excitation, as for ions excited to relativistic speed and/or ions in a nonquadrupolar trapping potential.

Axial dipolar excitation [excitation voltages applied differentially between the two trapping ("end cap") electrodes (Figure 1f)] has been used for the detection of stored ions by a direct observation of their coherent trapping motion (in that case, with a hyperbolic trap) [11]. Because the trapping frequency is significantly lower than the ion cyclotron frequency, axial dipolar excitation provides a useful means for resonant (i.e., mass selective) ejection of electrons [18] or unwanted low-mass ions whose high ion cyclotron frequencies might exceed the bandwidth of the excitation frequency synthesizer and/or amplifier, as previously demonstrated in an ICR "drift" cell [19].

By parametric excitation (detection) we mean an excitation (detection) geometry that is identical to the trapping geometry; that is, the conventional trapping electrodes are combined in one set, all of the remaining electrodes are combined in a second set, and the excitation signal is applied to (the image charge is picked up from) these sets (Figure 1g). The parametric mode operation was introduced to ICR by Lee et al. [7] who used a cylindrical trap. Parametric mode FT-ICR has since been performed with a hyperbolic trap [20]. In both cases, ions were produced off-axis before excitation. Recently, a parametric mode excitation technique has been applied to ions created initially on-axis and subsequently shifted off-axis by excitation at their magnetron frequency; the ion signal was then observed in the conventional dipole fashion at the reduced cyclotron frequency, $\omega_{+}=\omega_{\mathrm{c}}-\omega_{-}$[12]. It has also been pointed out [12] that only two electrodes are required for ion trapping; excitation and detection in such a two-electrode trap may both be performed in the parametric mode. The construction and performance of such a trap have recently been reported [21].

Radial ( $x y$ ) quadrupolar excitation, with an excitation voltage of one phase applied equally to one pair of opposed side electrodes and an excitation voltage of opposite phase applied equally to the other pair of opposed side electrodes (Figure 1h), has two uses. Such excitation at frequency $2 \omega_{+}$is shown to lead to an exponential increase in ion cyclotron orbital radius, whereas the same excitation performed at frequency $\omega_{+}+\omega_{-}=\omega_{c}$ produces periodic interconversion between cyclotron and magnetron motions. The latter effect has been exploited extensively in ICR determination of masses of short-lived isotopes at ISOLDE/
CERN [22], providing not only high-accuracy mass determination of the ions under investigation [23], but also cooling of the ions by adding a buffer gas to introduce ion-neutral collisions during the quadrupolar excitation period [24]. Radial quadrupolar excitation has recently been adapted to FT-ICR experiments [25], where it is used to axialize initially off-axis ions for extended trapping periods at high pressure and/or improved detection efficiency. Radial quadrupolar ( $x y$ ) excitation is closely related to quadrupolar $(x z)$ excitation, very recently introduced to FT-ICR [26], in which the trapping and one pair of side electrodes are segmented, so as to couple axial (trapping) ion motion to the two radial (cyclotron and magnetron) ion motions.

\section{Theory}

\section{Ion Motion and Energy}

In the absence of an applied time-varying electric field, the three independent motions of an ion trapped by a static magnetic field and a quadrupolar electrostatic field are well known [27]. Recently, various approaches to study these motions have been reviewed [28]. We therefore begin simply by stating the results. Parallel to the $z$ axis (i.e., the direction of the applied static magnetic field), the trapped ions perform a harmonic "trapping" oscillation (axial motion). In the $x y$ plane, the motion consists of a combination of two uncoupled circular modes, the cyclotron and the magnetron orbital motions. The ion position $\mathbf{r}$ and velocity $\mathbf{v}=\mathbf{i}$ (vectors are denoted by boldface symbols) are conveniently expressed in the form

$$
\begin{aligned}
& x=x_{+}+x_{-} \\
& y=y_{+}+y_{-} \\
& \dot{x}=\dot{x}_{+}+\dot{x}_{-} \\
& \dot{y}=\dot{y}_{+}+\dot{y}_{-}
\end{aligned}
$$

in which

$$
\begin{aligned}
& x_{+}=\rho_{+} \sin \left(\omega_{+} t\right) \\
& x_{-}=\rho_{-} \sin \left(\omega_{-} t\right) \\
& y_{+}=\rho_{+} \cos \left(\omega_{+} t\right) \\
& y_{-}=\rho_{-} \cos \left(\omega_{-} t\right) \\
& \dot{x}_{+}=\rho_{+} \omega_{+} \cos \left(\omega_{+} t\right) \\
& \dot{x}_{-}=\rho_{-} \omega_{-} \cos \left(\omega_{-} t\right) \\
& \dot{y}_{+}=-\rho_{+} \omega_{+} \sin \left(\omega_{+} t\right) \\
& \dot{y}_{-}=-\rho_{-} \omega_{-} \sin \left(\omega_{-} t\right)
\end{aligned}
$$

and

$$
\begin{aligned}
& z=z_{\max } \sin \left(\omega_{z} t\right) \\
& \dot{z}=z_{\max } \omega_{z} \cos \left(\omega_{z} t\right)
\end{aligned}
$$


in which $\rho_{+}, \rho_{-}$, and $z_{\max }$ are the instantaneous ion cyclotron orbital radius, magnetron radius, and trapping oscillation amplitude, respectively, and $\omega_{+}, \omega_{-}$, and $\omega_{z}$ are the reduced cyclotron, magnetron, and trapping frequencies, respectively. Figure 2 shows the magnetron and cyclotron orbits for arbitrary initial phases (i.e., arbitrary directions of $\rho_{+}$and $\rho_{-}$). In eq $2 \mathrm{a}-\mathrm{h}$, the initial phases have been set equal to zero for simplicity (i.e., $\rho_{+}$and $\rho_{-}$point along the positive $y$-axis in Figure 2). Explicitly,

$$
\begin{aligned}
& \omega_{+}=\frac{\omega_{\mathrm{c}}}{2}+\sqrt{\left(\frac{\omega_{\mathrm{c}}}{2}\right)^{2}-\frac{\omega_{z}^{2}}{2}} \\
& \omega_{-}=\frac{\omega_{\mathrm{c}}}{2}-\sqrt{\left(\frac{\left.\omega_{\mathrm{c}}\right)^{2}-\frac{\omega_{z}^{2}}{2}}{2}\right.} \\
& \omega_{z}=\sqrt{\frac{q V_{\text {trap }}}{m d_{0}^{2}}}
\end{aligned}
$$

in which

$$
\omega_{c}=q B / m
$$

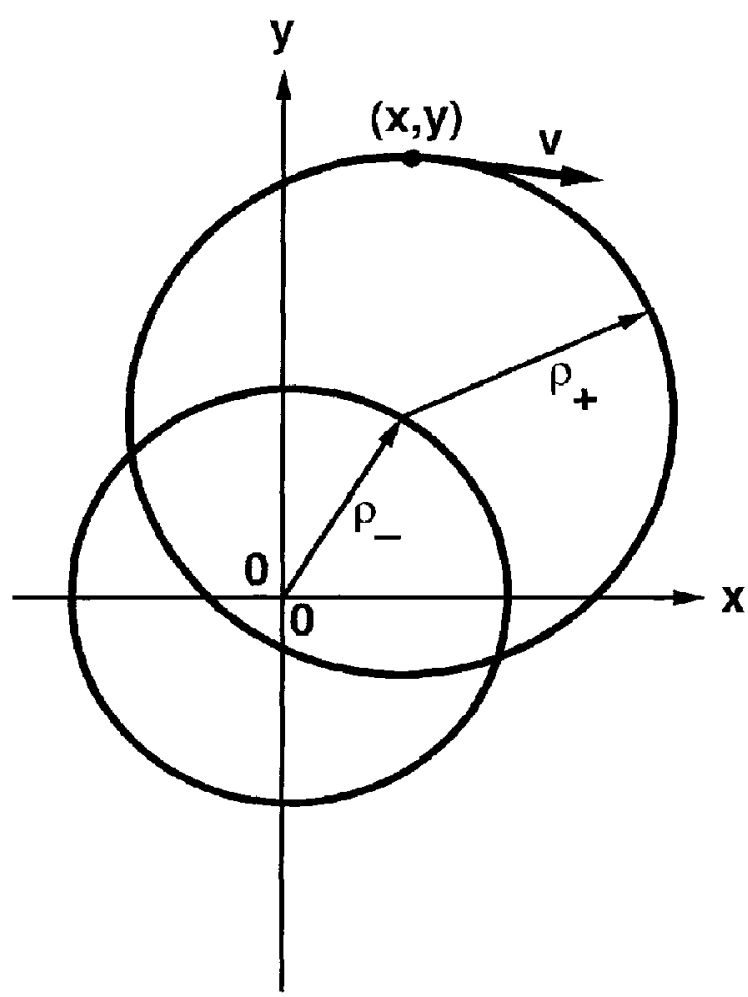

Figure 2. Magnetron (small circle of radius $\rho_{-}$) and cyclotron (large circle of radius $\rho^{-}$) orbits in a plane perpendicular to the applied magnetic field in the absence of RF electric field excitation. The center of the cyclotron orbit always lies on the magnetron circle (see Figure 3). is the unperturbed cyclotron frequency of an ion of mass $m$, and charge $q$ in a homogeneous magnetic field of amplitude $B$ (in the $z$ direction) in the absence of any electrostatic field; $V_{\text {trap }}$ is the trapping potential (e.g., between the trapping plates and the side plates for a cubic trap and between the ring and end cap electrodes for a hyperbolic trap); and $d_{0}$ is a measure of the trap dimensions. For a hyperbolic (Penning) trap [27], for example,

$$
d_{0}^{2}=\frac{1}{2}\left(\frac{\rho_{0}^{2}}{2}+z_{0}^{2}\right)
$$

in which $\rho_{0}$ and $z_{0}$ are the transverse and axial radii of the trap. For other trap configurations, eqs 4-6 still apply but with a different value of $d_{0}$. The trap potential near the center of a nunhyperbolic trap is approximately quadrupolar and may be evaluated by, for example, Taylor expansion techniques [3]. Although the electric field of a nonhyperbolic trap is not as nearly quadrupolar as that of the Penning trap, leading to such perturbations as coupling between the various ion modes to produce resonant responses to excitation at multiples and combinations (see below) of the frequencies of eqs 4-6, eqs 1-7 nevertheless give an approximate description of the ion motion in FT-ICR traps. (By reciprocity $[6,29,30]$, detection at multiples $[3,6,31]$ and combinations of the frequencies of eqs 4-6 also occurs in a nonquadrupolar trap potential.) For a cubic trap of edge length $a$, for example, one finds that [4]

$$
d_{0}^{2}=a^{2} / 2 \alpha, \quad \alpha=2.77373
$$

In the quadrupolar approximation, the three ion motional modes (cyclotron, magnetron, and trapping) are independent. Those amplitudes depend only on the ion initial position and velocity (and, of course, on the trap dimensions that ultimately limit the amplitude of each of the ion motions). For example, Figure 3 shows the radial ion motion (superposition of magnetron and cyclatron mode) for two ratios of cyclotron orbital radius to magnetron radkus in the absence of applied radio frequency (RF) excitation.

The following readily verified relationships [32] will prove useful later.

$$
\begin{aligned}
\omega_{+}+\omega_{-} & =\omega_{c} \\
\omega_{+} \omega_{-} & =\omega_{z}^{2} / 2 \\
\omega_{+}^{2}+\omega_{-}^{2}+\omega_{c}^{2} & =\omega_{c}^{2}
\end{aligned}
$$

The total energy $E_{\text {total }}$ of the ions in the trap can be separated into contributions from the cyclotron $\left(E_{+}\right)$, magnetron $\left(E_{-}\right)$, and trapping $\left(E_{T}\right)$ energy,

$$
E_{\text {total }}=E_{+}+E_{-}+E_{T}
$$



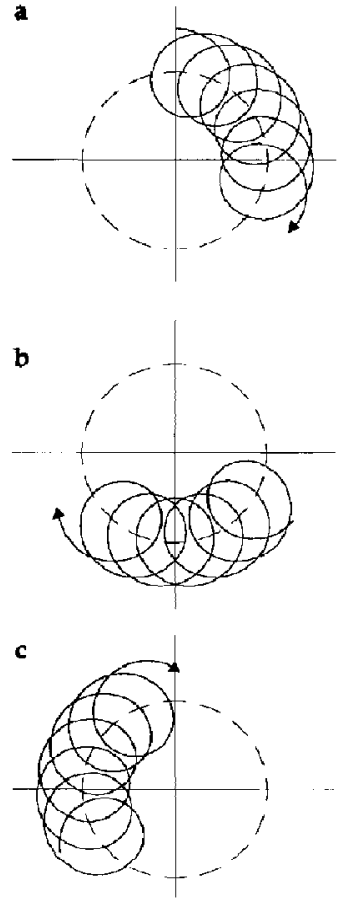

a
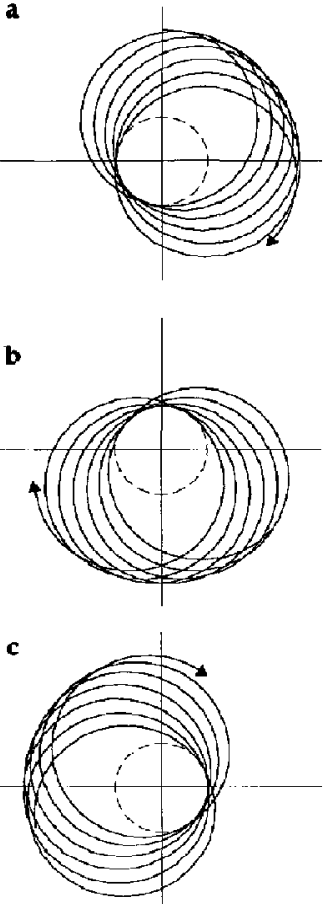

Figure 3. Radial ion motions in an FT-ICR trap, in the absence of RF electric field excitation (see eqs 2, 4, and 5). Dashed circles magnetron orbit of radius $\rho_{-}$(i.e., the trajectory of the instantaneous center of the ion cyclotron orbital motion of radius $\rho_{4}$ ); $\omega_{+} / \omega_{-}=20$. Top, $0 \leq \omega_{+} \mathrm{t} \leq 40 ;$ middle, $40 \leq \omega_{+} t \leq 80 ;$ bottom. $80 \leq \omega_{+} t \leq 120 ;$ left. $\rho_{+}=\rho_{-} / 2 ;$ right, $\rho_{+}=2 \rho \ldots$.

I Jere the energy of each mode represents the sum of its respective kinetic and potential energy and can be expressed in terms of the motional amplitudes ( $\rho_{+}$, $\rho_{-}$, and $z_{\max }$ ) or in terms of their quantum numbers $n_{+}, n_{-}$, and $n_{2}[27]$ :

$$
\begin{aligned}
E_{+} & =\frac{m}{2}\left(\omega_{+}^{2}-\omega_{+} \omega_{-}\right) \rho_{+}^{2} \\
& =\frac{m}{2} \omega_{\mathrm{p}} \omega_{+} \rho_{+}^{2}=\left(n_{+}+\frac{1}{2}\right) \frac{h}{2 \pi} \omega_{+} \\
F_{-} & =\frac{m}{2}\left(\omega_{-}^{2}-\omega_{+} \omega_{-}\right) \rho_{-}^{2} \\
& =-\frac{m}{2} \omega_{\mathrm{p}} \omega_{-} \rho_{-}^{2}=-\left(n_{-}+\frac{1}{2}\right) \frac{h}{2 \pi} \omega_{-} \\
E_{T} & =\frac{m}{2} \omega_{z}^{2} z_{\max }^{2}=\left(\mathrm{n}_{\mathrm{z}}+\frac{1}{2}\right) \frac{\mathrm{h}}{2 \pi} \omega_{\mathrm{z}}
\end{aligned}
$$

in which

$$
\omega_{p}=\omega_{+}-\omega_{-}=\omega_{c}-2 \omega_{-}
$$

is the frequency of parametric excitation and/or detection (see below) $[7,12,20,33]$, and $h$ is Planck's

constant. From eq 17 , eq 12 can be rewritten as

$$
\omega_{p}^{2}=\omega_{+}^{2}+\omega_{-}^{2}-\omega_{z}^{2}
$$

and the following relation is readily derived:

$$
\omega_{c}^{2}+\omega_{p}^{2}=2\left(\omega_{+}^{2}+\omega_{-}^{2}\right)
$$

Note that the magnetron mode energy is always negative and decreases with growing quantum number (i.e. decreases with increasing magnetron radius) because the potential energy predominates over the kinetic energy for magnetron motion.

\section{Ion Motional Amplitudes from Instantaneous Power Absorption: Method and Assumptions}

In the following derivations, we assume that the various ion motions are approximately the same during one motional cycle (cyclotron, magnetron, or trapping) in the presence or absence of an applied electric excitation field. In other words, the excitation field is sufficiently weak that the energy absorbed per motional cycle is small compared to the energy of the motion itself. This approximation is similar to the recently published perturbation treatment of Mitchell [34]. With this simplification, the ion motion during one cycle of a given motional mode may still be described by eqs 1-7. The instantaneous power absorption $P(t)$, is then simply

$$
P(t)=\mathbf{F} \cdot \mathbf{v}
$$

in which $\mathbf{v}$ is the ion velocity, and $\mathbf{F}$ is the excitation force acting on the ion,

$$
\mathbf{F}=q \mathbf{E}
$$

and $\mathbf{E}$ is the excitation electric field

$$
\mathbf{E}=\boldsymbol{\alpha}(\mathbf{r}) \sin (\omega t+\phi)
$$

in which $\alpha(\mathbf{r}), \omega$, and $\phi$ are the amplitude, frequency, and phase of $\mathbf{E}$, respectively. 'Ihe excitation field amplitude, $\boldsymbol{\alpha}(\mathbf{r})$ is in general a function of the ion position r. In this study, we consider only harmonic (singlefrequency resonant) excitation, so that $\omega$ is chosen to match the frequency of one (or a combination) of the ion natural motional modes.

The instantaneous power absorption is then obtained directly from eq 20 , by specifying the appropriate excitation frequency (e.g., $\omega=\omega_{+}$or $\omega_{-}$or $\omega_{\mathrm{T}}$ from eqs 4-6) and excitation field amplitude $\alpha(r)$ and the appropriate ion velocity expressions (eq $2 \mathrm{e}^{-\mathrm{h}}$; eq $3 b)$. We then average the instantaneous power absorption over one cycle of the specified excitation frequency (see below) and equate the result to the time derivative of the energy (eqs 14-16) of the ion mode(s) being excited (eqs 14-16) to obtain a differential equa- 
tion for the time rate of change of the ion motional amplitude of interest [e.g., $\rho_{+}(t), \rho_{-}(t), z_{\max }(t)$ ]. We then solve the differential equation, subject to appropriate initial conditions, to yield the (time dependent) ion motional amplitude [e.g., $\rho_{+}(t), \rho_{-}(t), z_{\max }(t)$ ] during the excitation process.

\section{Standard Dipolar Radial Excitation}

Here we rederive the previously known result for dipolar radial ("azimuthal") excitation to illustrate the method and notation before proceeding to other excitation geometries. [We shall consider dipolar axial ("polar") excitation in a later section.] For dipolar radial excitation, the amplitude $\boldsymbol{\alpha}(\mathrm{r})$ of the dipolar radial excitation electric field is independent of position $\mathbf{r}$

$$
\mathbf{\alpha}=\left(E_{0}, 0,0\right)
$$

For example, in the infinite parallel plate approximation $[3,35]$,

$$
\boldsymbol{\alpha}=\left(V_{\mathrm{p}-\mathbf{p}} / d, 0,0\right)
$$

in which $V_{\mathrm{p}-\mathrm{p}}$ is the peak-to-peak amplitude of the differentially applied excitation voltage, and $d$ is the separation between the two flat excitation electrodes. Similarly, near the center of a cubic trap of edge length $a$, the excitation field can be approximated as

$$
\alpha=\left(\beta V_{\mathrm{p}-\mathrm{p}} / a, 0,0\right)
$$

in which $\beta=0.72167$ [4]. Equation 25 applies to other trap geometries as well, provided that the appropriate value of $\beta$ is used. We shall therefore use the $\beta V_{\mathrm{p}-\mathrm{p}} / a$ expression in this and succeeding sections.

Cyclotron Orbital Motion (Quadrupolar Trapping Potential). We may now substitute $F=q E$ (with $\alpha$ from eq 25) and $\mathbf{v}=\left(\dot{x}_{+}+\dot{x}_{-}\right) \mathbf{i}+\left(\dot{y}_{+}+\dot{y}_{-}\right) \mathbf{j}$ (with $\dot{x}_{+}, \dot{x}_{-}$, $\dot{y}_{+r}$ and $\dot{y}_{-}$from eqs $2 \mathrm{e}-\mathrm{h}$ ) into eq 20 , to obtain the instantaneous power, $\mathrm{P}(\mathrm{t})$, absorbed by the ions:

$$
\begin{aligned}
P(t)= & \left(q \beta V_{\mathrm{p}-\mathrm{p}} f a\right) \sin (\omega t+\phi)\left[\rho_{+} \omega_{+} \cos \left(\omega_{+} t\right)\right. \\
& \left.+\rho_{-} \omega_{-} \cos \left(\omega_{-} t\right)\right]
\end{aligned}
$$

The maximum power absorption for ion cyclotron orbital motion (averaged over a time interval that is long compared to one period of the cyclotron motion) occurs for $\phi=\pi / 2$ and $m=\omega_{+}$(i.e., resonant excitation at the ICR orbital frequency), with $90^{\circ}$ difference between excitation electric field phase and ion cyclotron orbital phase. For an excitation of duration $T$, equal to one period of the ion cyclotron orbital motion, we may replace $P(t)$ by its time-averaged value,

$$
\begin{gathered}
\langle P\rangle=\frac{1}{T} \int_{0}^{T} P(t) d t \approx \frac{q \beta V_{\mathrm{p}-\mathrm{p}} \rho_{+} \omega_{+}}{2 a} \\
\text { for } T=2 \pi / \omega_{+}
\end{gathered}
$$

From here on, we shall denote the average power absorption $\langle P\rangle$ simply as $P$, to indicate that a time average has been taken. The instantaneous power absorption will continue to be represented by $P(t)$.

Alternatively, the power absorption leading to increased ion cyclotron orbital energy may be represented as the time rate of change of the cyclotron orbital energy given by eq 14 :

$$
P=d E_{+} / d t=m \omega_{\mathrm{p}} \omega_{+} \rho_{+} \dot{\rho}_{+}
$$

'Ihus, by equating eqs 27 and $28 a$, we obtain a differential equation for the time evolution of the ion $\mathrm{cy}$ clotron orbital radius $\rho_{+}$:

$$
m \omega_{\mathrm{p}} \dot{\rho}_{+}=\frac{q \beta V_{\mathrm{p}-\mathrm{p}}}{2 a}
$$

The solution for this differential equation [where for simplicity we take the initial cyclotron orbital radius to be zero, $\left.\rho_{+}(0)=0\right]$ is

$$
\rho_{+}(t)=\frac{q \beta V_{\mathrm{p}-\mathrm{p}} t}{2 m a \omega_{\mathrm{p}}}
$$

Figure 4 shows the radial ion motion during resonant
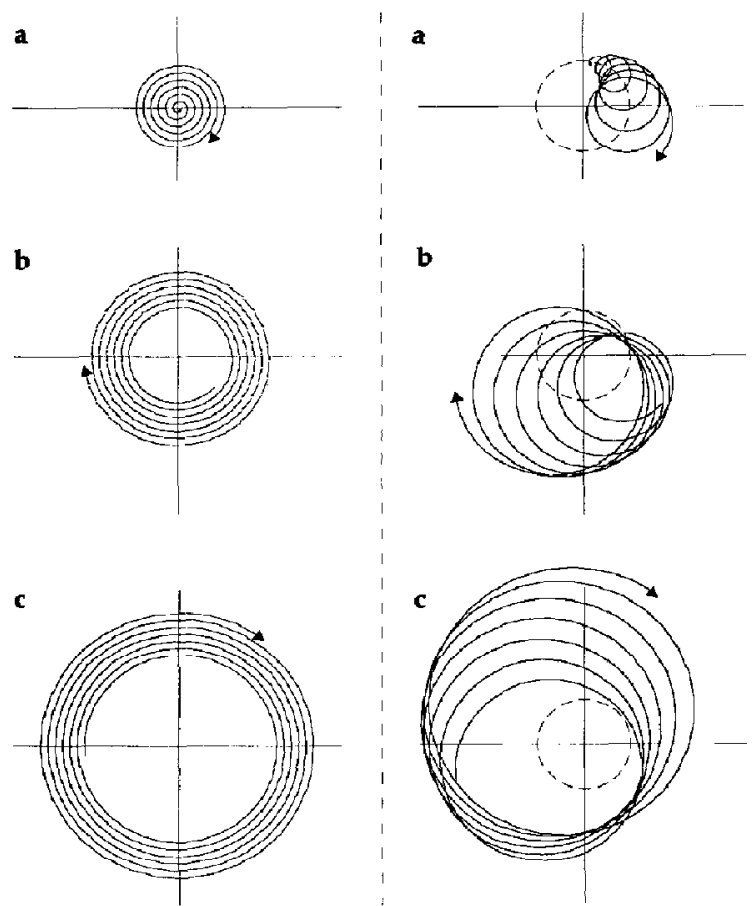

Figure 4. Radial ion motion for standard dipolar radial excitation at the ion cyclotron orbital frequency $\omega_{4}$ in a quadrupolar electrostatic trapping potential (see eq 30). Left, ion initially at rest in the center of the trap (zero initial magnetron radius); right, nonzero initial magnetron radius. Dashed circles, frequency ratio, and time periods as in Figure 2. 
cyclotron excitation for an ion initially at rest at the center of the trap. The ion trajectory is an Archimedes spiral, that is, the distance between the lines of the trajectory of consecutive revolutions remains constant (in contrast to, e.g., the feedback excitation discussed below). It should be noted that the time averaging used in this derivation leads to a slightly simplified ion trajectory. The actual trajectory includes an additional oscillation of the instantaneous center of the cyclotron motion (i.e., an excitation/deexcitation of the magnetron motion) [36]; however, this effect is negligible within the limit that the excitation lasts for many periods of the ion cyclotron orbital frequency $\omega_{1}$, as assumed above.

Cyclotron Orbital Motion (Zero Trapping Potential). In the absence of any electrostatic trapping field, the preceding calculation proceeds along similar lines, with the following differences. First, the cyclotron orbital motion is purely kinetic (i.e., zero magnetron motion because there is no electrostatic potential), and the cyclotron orbital energy from eq 14 simplifies $\left(\omega_{\mathrm{p}}=\right.$ $\left.\omega_{+}-\omega_{\mathrm{c}}\right)$ to

$$
E_{\mathrm{c}}=\frac{m}{2} \omega_{\mathrm{c}}^{2} \rho_{\mathrm{c}}^{2}
$$

in which $\rho_{\mathrm{c}}=\rho_{+}$is the ion cyclotron orbital radius. The power absorbed (eq 28), then simplifies to

$$
P=m \omega_{c}^{2} \rho_{\mathrm{c}} \dot{\rho}_{\mathrm{c}}
$$

which leads to the differential equation

$$
m \omega_{\mathrm{c}} \dot{\rho}_{\mathrm{c}}=\frac{q \beta V_{\mathrm{p}-\mathrm{p}}}{2 a}
$$

and its solution

$$
\rho_{\mathrm{c}}(t)=\frac{q \beta V_{\mathrm{p}-\mathrm{p}} t}{2 m a \omega_{c}}
$$

(in which the same assumptions for phase coherence and initial conditions have been applied as in the quadrupolar trapping potential case). After substituting for $\omega_{c}=q B / m$, eq $30 \mathrm{~b}$ takes the familiar form,

$$
p_{\mathrm{c}}(t)=E_{0} t / 2 B
$$

Because $\omega_{c} \geq \omega_{\mathrm{p}}$ (compare eqs 10 and 17), a comparison of eqs $30 \mathrm{a}$ and $b$ reveals that the cyclotron orbital radius increases more rapidly in the presence than in the absence of the quadrupolar electrostatic trapping potential. As the ion cyclotron orbital radius increases during excitation, the ion absorbs the same amount of energy from the excitation field in the presence or absence of an electrostatic trapping potential; however, in the presence of a quadrupolar trapping potential, the ion slides downhill along the elec- trostatic potential energy surface as it moves radially outward and thereby gains additional kinetic energy (beyond that due to the excitation itself) equal to that decrease in potential energy.

\section{Magnetron Motion (Quadrupolar Trapping Potential)}

Now suppose that we excite at the magnetron frequency $\omega_{-}$rather than the cyclotron orbital frequency $\omega_{1}$ in eq 26. Following the same procedure as before, we find that for maximum power absorption (again $\phi=\pi / 2$ ), eqs $27,28 \mathrm{a}$, and $\mathrm{b}$ now take the form

$$
\begin{aligned}
& P=\frac{q \beta V_{\mathrm{p}-\mathrm{p}} \rho_{-} \omega_{-}}{2 a} \\
& P=\frac{d E_{-}}{d t}=-m \omega_{\mathrm{p}} \omega_{-} \rho_{-} \dot{\rho}_{-}
\end{aligned}
$$

leading to a differential equation for the magnetron radius $\rho_{-}(t)$,

$$
-m \omega_{\mathrm{p}} \dot{\rho}_{-}=\frac{q \beta V_{\mathrm{p}-\mathrm{p}}}{2 a}
$$

whose solution [for an initial magnetron radius $\boldsymbol{\rho}_{-}(0)$ at time zero] is

$$
\rho_{-}(t)=\rho_{-}(0)-\frac{q \beta V_{\mathrm{p}-\mathrm{p}} t}{2 m a \omega_{\mathrm{p}}}
$$

Equation 35 indicates that resonant dipolar radial excitation decelerates the magnetron motion of an initially off-axis ion [i.e., $\rho_{-}(0) \neq 0$ ] if the ion magnetron motion and the excitation electric field differ in phase by $\phi=\pi / 2$ (i.e., maximum power absorption, as in the prior case of cyclotron orbital motion); however, acceleration of the magnetron motion of an off-axis ion occurs for $\phi=-\pi / 2$. An ion initially on-axis in the trap $\left[\rho_{-}(0)=0\right]$ will increase its magnetron radius linearly with time (leading eventually to radial ejection) during the excitation event. In fact, by observing a conventional FT-ICR time-domain signal following excitation at the ion magnetron frequency, such ejection can be detected to provide a direct method for determination of the magnetron frequency [2]. It is not necessary to eject the ions during the magnetron excitation event. If the sum of the magnetron and cyclotron radii (after subsequent excitation of the cyclotron motion) exceeds the trap dimensions, the ion signal disappears, indicating that the prior magnetron excitation was resonant. This scheme is very similar to those in which the ion number is monitored by axial ejection of the ions through small holes in the trapping plates, followed by ion counting with multichannel plate detectors [37].

Equation 35 also shows that it is advisable not to include the ion magnetron frequency in the range of 
broadband (chirp [38, 39] or stored waveform inverse Fourier transform (SWIFT) $[4,40,41])$ excitation waveforms. It is worth noting that direct current (DC) "Dirichlet" ("impulse") [15, 17, 42] or DC "burst" [43] excitation necessarily excites low-frequency magnetron motion because a Fourier transform of a time-domain DC signal of duration $T$, yields a frequency-domain "sinc" spectrum of the form $\sin (\omega T) / \omega$ [17]. From eqs $30 \mathrm{a}$ and 35 , it is clear that the ion cyclotron orbital radius following such a DC "impulse" excitation is always smaller than the post-excitation magnetron radius (provided that the ion was at rest in the center of the trap before the excitation event) because $\omega_{-} \leq \omega_{+}$, and the excitation "sinc" amplitude decreases with increasing frequency. Finally, there is no analog to eq $30 \mathrm{~b}$ because magnetron motion no longer exists in the absence of an electrostatic trapping potential.

Having illustrated the formalism of our approach with the well-known dipolar radial excitation example, we now proceed to treat in the same way various other excitation modes, some of which have not been previously analyzed. The method in each case remains the same; only the form of $\mathrm{E}$ and the ion motional modes of interest vary.

\section{Quadrature Excitalion}

In quadrature excitation, oscillating electric fields of the same frequency (but different phase) are applied to both pairs of side plates of, for example, a cubic trap (Figure 1d). The electric field takes the form

$$
\mathbf{E}=\frac{\beta V_{\mathrm{p}-\mathrm{p}}}{d}\left(\sin \left(\omega t+\phi_{1}\right), \sin \left(\omega t+\phi_{2}\right), 0\right)
$$

and the instantaneous power absorption (eq 20) becomes

$$
\begin{aligned}
P(t)=\mathbf{F} \cdot \mathbf{v}= & \frac{q \beta V_{\mathrm{p}-\mathrm{p}}}{d}\left\{\sin \left(\omega t+\phi_{1}\right)\right. \\
& \times\left[\rho_{+} \omega_{-} \cos \left(\omega_{+} t\right)+\rho_{-} \omega_{-} \cos \left(\omega_{-} t\right)\right] \\
& -\sin \left(\omega t+\phi_{2}\right)\left[\rho_{+} \omega_{+} \sin \left(\omega_{+} t\right)\right. \\
& \left.\left.+\rho_{-} \omega-\sin \left(\omega_{-} t\right)\right]\right\}
\end{aligned}
$$

For in-phase single-frequency on-resonance excitation of ion cyclotron motion, $\phi_{1}=\pi / 2$, and $\phi_{2}=\pi$ (i.e., the electric field is circularly polarized, as opposed to the linearly polarized dipole field discussed above), and $\omega=\omega_{+}$. The power absorption averaged over one cycle of $\omega_{+}$then becomes

$$
P=\frac{q \beta V_{\mathrm{p}-\mathrm{p}} \rho_{+} \omega_{+}}{d}
$$

Note that twice as much power is absorbed for circularly polarized radial excitation (eq 38) as for standard linearly polarized dipolar excitation (eq 27). The reason is simply that a linearly polarized electric field $E_{0}$ $\sin (\omega t)$ may be constructed as the sum of two counterrotating circularly polarized electric fields,

$$
E_{0} \sin (\omega t)=\frac{E_{0}}{2} \exp (i \omega t)+\frac{E_{0}}{2} \exp (-i \omega t)
$$

of which only the field rotating in the same sense as the ion has a significant effect $[16,17]$.

By equating eq 38 to the time derivative of the cyclotron energy (eqs 28a and b), we obtain a differential equation from which the ion cyclotron orbital radius $p_{+}(t)$ is readily shown to be

$$
\rho_{+}(t)=\frac{y \beta V_{\mathrm{p}-\mathrm{p}} l}{m d \omega_{\mathrm{p}}}
$$

In the absence of an electrostatic trapping potential, the same steps that led from eq $30 \mathrm{a}$ to $\mathrm{b}$ yield the ion cyclotron orbital radius resulting from quadrature excitation:

$$
\rho_{\mathrm{c}}(t)=\frac{q \beta V_{\mathrm{p}-\mathrm{p}} t}{m d \omega_{\mathrm{c}}}
$$

Quadrature excitation has two advantages over dipolar excitation: (1) only half the excitation voltage magnitude is required to excite an ion to a given ion cyclotron orbital radius; and (2) it is possible to excite selectively ions of a given charge sign (because ions of the opposite charge sign rotate in the opposite sense), as recently demonstrated [16] for simultaneously trapped ions in an ICR ion trap with RF trapping potential [44]. Similarly, signal to noise ratio is en hanced by a factor of $2 / \sqrt{2}=\sqrt{2}$ for quadrature detection compared with linear dipolar detection [45-47].

\section{Feedback Excitation}

One way of ensuring that the excitation frequency remains on-resonance is to set it equal to the frequency of the detected ICR signal of the ions themselves, taking into account any phase difference between the detected signal and the transmitted excitation. For example, the ICR signal from a coherently orbiting ion packet in a cubic trap of edge length $a$ could be detected on the electrodes at $y= \pm a / 2$ and then transmitted after amplification to the electrodes at $x=$ $\pm a / 2$. In a predominantly capacitive detector $[35,48]$, the ion signal is proportional to the number of ions and their $y$-displacement [35, 48, 49]. In general, phase-shifting devices could be used to match the phases of excitation and detection signals (Figure 1e). This excitation scheme has not yet been realized experimentally, partly because the transmission and detection circuits have to be extremely well isolated to prevent the excitation signal from coupling directly to the detector. The feasibility of the method might be 
improved by exciting and detecting at different harmonics or combination frequencies. We shall assume that the detection bandwidth does not extend to the magnetron frequency, so that the excitation signal is of the form,

$$
\mathrm{E}=(1,0,0)(A / N) \rho_{+} \sin \left(\omega_{+} t+\phi\right)
$$

in which $A$ is a proportionality factor that includes the amplification factor of the feedback loop as well as the number of ions of reduced cyclotron frequency at the frequency of interest. From eq 20, the power absorption averaged over one cycle of $\omega_{+}$becomes $(\phi=\pi / 2$ for maximal response)

$$
P=\frac{q A \rho_{+}^{2} \omega_{+}}{2}
$$

Equating eq 42 with eq 28 for the time rate of change of cyclotron orbital energy, we obtain

$$
P=m \omega_{\mathrm{p}} \omega_{+} \rho_{+} \dot{\rho}_{+}=\frac{q A \rho_{+}^{2} \omega_{+}}{2}
$$

from which we obtain the differential equation for $\rho_{+}$

$$
m \omega_{\mathrm{p}} \dot{\rho}_{+}=\frac{q A \rho_{+}}{2}
$$

whose solution is simply the exponential function

$$
\rho_{+}(t)=\rho_{+}(0) \exp \left(\frac{q A}{2 m \omega_{p}} t\right)
$$

For the ions not to be ejected from the trap, the amplification factor $A$ could be varied with input (ICR signal) amplitude so that the excitation terminates once a specified ICR signal magnitude is reached. It is worth noting that feedback excitation may prove difficult experimentally. Apart from the problem of isolating excitation from detection, the feedback signal depends on the number of ions, with two consequences. First, the cyclotron radius of the excited ion packet must be measured or calculated to determine the number of ions $[3,6,49,50]$ in the proportionality factor $A$. Second, if ions of more than one mass-to-charge ratio are present, then the excitation factor may be different for ions of each mass-to-charge ratio if their abundances are different (which is generally the case). $\mathrm{Nev}^{-}$ ertheless, feedback excitation could be useful when the ion motional frequency depends strongly on ion position. For example, light and /or multiply charged ions have high ion cyclotron orbital frequencies [51]. Even at a relatively small ion cyclotron orbital radius, the ion cyclotron orbital frequency can shift by more than $1 \mathrm{kHz}$ owing to the relativistic increase in mass due to increased ion velocity [52]. In such a case, continuously on-resonance excitation could be achieved by an initial resonant $\mathrm{RF}$ "impulse" $[15,17]$ or "burst" [43] dipolar radial excitation followed by the proposed feedback excitation. (Alternatively, it is possible to calculate analytically the excitation phase that exactly matches the ion cyclotron orbital phase during the excitation process [53].) Finally, the feedback excitation method could in principle be applied to ions of different mass-to-charge ratios and of different relative abundance.

\section{Frictional Damping}

The ion trajectory described by the quadrature equivalent of eq 45 is the time inverse of the trajectory resulting from a damping force on a pure ion cyclotron orbital motion (Figure 5). Consider a frictional resistive force proportional to the ion speed, for example, due to collisional damping of the ion motion,

$$
\mathbf{F}=-f \mathbf{v}
$$

in which $f$ is the frictional coefficient [54]. Such frictional damping might arise, for example, from collisions of high-mass ions with low-mass neutrals. The damping is equivalent to a negative feedback quadrature excitation of the radial ion motions plus an additional negative feedback excitation of the axial ion motion. The total power absorbed by the ion is

$$
P(t)=\mathbf{F} \cdot \mathbf{v}=-f v^{2}
$$

Because the total effective damping force is a linear combination of dipolar "deexcitations" at $\omega_{+}, \omega_{-}$, and $\omega_{z}$, with the appropriate spatial dependence, there is no coupling among the various ion motional modes (in contrast to parametric and quadrupolar radial excitation - see below), and the motional modes may be considered one at a time. The time-averaged power absorption is readily obtained from eq 47 , with $\mathrm{v}_{x}, v_{y}$, and $v_{z}$ from eq $2 \mathrm{e}-\mathrm{h}$ and eq $3 \mathrm{~b}$ :

$$
\begin{aligned}
& P_{+}=-f \omega_{+}^{2} \rho_{+}^{2} \\
& P_{-}=-f \omega_{-}^{2} \rho_{-}^{2} \\
& P_{z}=-f \omega_{z}^{2} z_{\max }^{2} / 2
\end{aligned}
$$

The corresponding time rate of change of energy for each of the three ion motional modes is

$$
\begin{aligned}
& d E_{+} / d t=m \omega_{p} \omega_{+} \rho_{+} \dot{\boldsymbol{\rho}}_{+} \\
& d E_{-} / d t=-m \omega_{p} \omega_{-} \rho_{-} \dot{\boldsymbol{\rho}}_{-} \\
& d E_{z} / d t=m \omega_{z}^{2} z_{\max } \dot{z}_{\max }
\end{aligned}
$$

Equating eq $48 a$ (or eq $48 b$ or c) to eq $49 a$ (or eq $49 b$ or 
$\mathbf{a}$

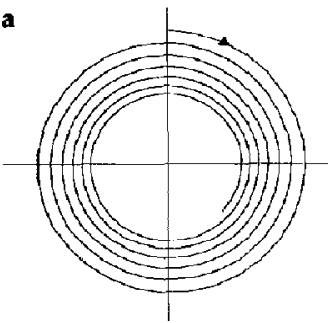

b
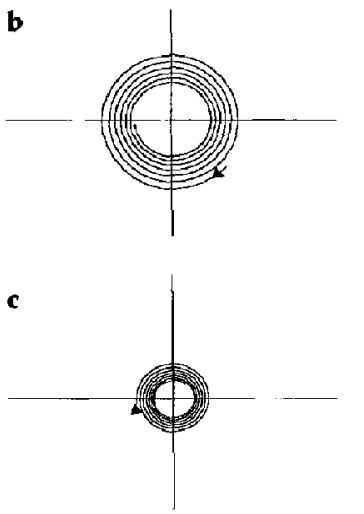

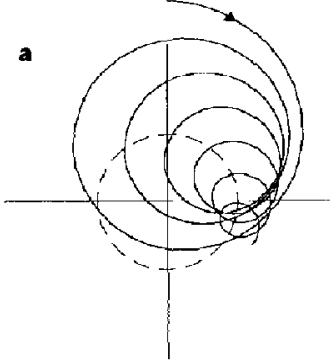

b
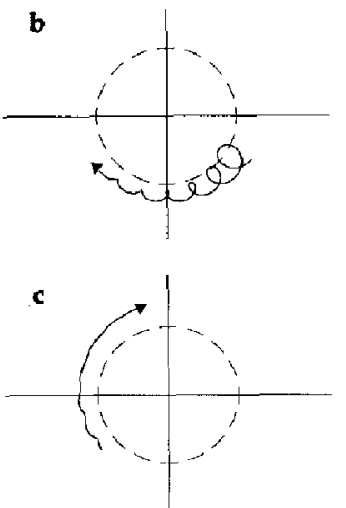

Figure 5. Damping of radial ion motions by a frictional force (see eq 51); $\omega_{+} / \omega_{-}=20$. Left, zero initial magnetron radius: the ion cyclotron orbital radius decreases exponentially to zero. Right, nonzero initial magnetron radius; the ion cyclotron orbital radius decreases exponentially to zero, but the magnetron radius increases exponentially with time. Dashed circles, initial magnetron orbital circle (i.e., the instantaneous position of the center of the ion cyclotron orbital motion if the magnetron radius were constant).

c) leads to the differential equations

$$
\begin{gathered}
\dot{\rho}_{+}=-\frac{f \omega_{+}}{m \omega_{\mathrm{p}}} \rho_{+} \\
\dot{\rho}_{-}=+\frac{f \omega_{-}}{m \omega_{\mathrm{p}}} \rho_{-} \\
\dot{z}_{\max }=-\frac{f}{2 m} z_{\max }
\end{gathered}
$$

whose solutions are

$$
\begin{array}{r}
\rho_{+}(t)=\rho_{+}(0) \exp \left(-\frac{f \omega_{+}}{m \omega_{\mathrm{p}}} t\right) \\
\rho_{-}(t)=\rho_{-}(0) \exp \left(+\frac{f \omega_{-}}{m \omega_{\mathrm{p}}} t\right) \\
z_{\text {max }}(t)=z_{\text {max }}(0) \exp \left(-\frac{f}{2 m} t\right)
\end{array}
$$

Figure 5 shows the effect of a damping force on the radial ion motion. If the ions start out with a pure cyclotron orbital motion (e.g., after resuriant dipolar radial excitation at their reduced ion cyclotron orbital frequency $\omega_{+}$, they simply spiral back to the center of the trap (Figure 5, left) and are available for another excitation [55]. If, however, the ion packet is initially displaced from the $z$ axis (i.e., nonzero initial magnetron radius), then that magnetron radius will increase owing to frictional damping, and the ions will eventually be lost from the trap (Figure 5, right).

Power absorption by the detector circuit can also lead to an increase in the ion magnetron radius and eventually to the loss of ions from the trap, as has recently been considered by Beu and Laude [56]. The present treatment includes both collisional damping and resistive damping from the detector circuit.

\section{Dipolar Excitation of Axial Motion}

In the case of an axial dipolar excitation (Figure 1f), the $\mathrm{RF}$ excitation electric field takes the form

$$
\mathbf{E}=\left(0,0, E_{0}\right) \sin (\omega t+\phi)
$$

The instantaneous power absorption (see eq 20) is

$$
P=\mathbf{F} \cdot \mathbf{v}=q E_{0} \sin (\omega t+\phi) z_{\max } \omega_{z} \cos \left(\omega_{z} t\right)
$$

Maximal on-resonance power absorption occurs for $\phi=\pi / 2$ and $\omega=\omega_{z}$. Averaged over one cycle of $\omega_{z}$ the power absorption becomes

$$
P=\frac{q E_{0} z_{\max } \omega_{2}}{2}
$$

Equating eq 54 to the time rate of change of ion axial energy (see eq 16),

$$
P=d E_{z} / d t=m \omega_{z}^{2} z_{\max } \dot{z}_{\max }
$$

leads to the differential equation

$$
m \omega_{z} \dot{z}_{\max }=\frac{q E_{0}}{2}
$$

The amplitude of trapping motion therefore evolves as

$$
z_{\max }(t)=\frac{q E_{0} t}{2 m \omega_{z}}
$$

In contrast to the evolution of the cyclotron radius discussed above, $z_{\max }(t)$ depends on the mass of the ion and the trapping voltage:

$$
z_{\max }(t)=\frac{E_{0} t}{2} \sqrt{\frac{q d_{0}^{2}}{m V_{\text {trap }}}}
$$


For a cubic trap (eqs 9 and 25),

$$
\begin{aligned}
\mathbf{E} & =\left(0,0, E_{0}\right) \sin (\omega t+\phi) \\
& =\left(0,0, \beta V_{\mathrm{p}-\mathrm{p}} / a\right) \sin (\omega t+\phi)
\end{aligned}
$$

and the procedure starting with eq 53 can be repeated (see eq 9) to yield

$$
z_{\max }(t)=\frac{\beta V_{\mathrm{p}-\mathrm{p}} t}{2} \sqrt{\frac{q}{2 \alpha m V_{\mathrm{tA \lambda} \mathrm{p}}}}
$$

in which, again, $\alpha=2.77373$ and $\beta=0.72167$. Note that $z_{\max }(l)$ is independent of the size of the cubic trap.

\section{Parametric Excitation of Axial Motion}

We turn next to parametric excitation (Figure 1g), for which the spatial dependence of the excitation electric field is the same as that of the trapping field. We begin as usual from a harmonic excitation signal,

$$
\begin{aligned}
& \mathbf{E}=(x, y,-2 z) \frac{V_{\mathrm{p}-\mathrm{p}}}{2 d_{0}^{2}} \sin (\omega t+\phi) \\
& \mathbf{E}=(x, y,-2 z) \frac{V_{\mathrm{p}-\mathrm{p}} \alpha}{a^{2}} \sin (\omega t+\phi) \quad \text { (cubic trap) }
\end{aligned}
$$

However, because parametric FT-ICR excitation has to date been used experimentally only with hyperbolic traps (see, however, the next section for radial motions) [11,20], only eq 61a is developed in this and the next section. The instantaneous power absorbed (eqs $3 \mathrm{~b}, 20,61$ ) by the axial (trapping) motion is

$$
\begin{aligned}
P(t)= & -2 z_{\max } \sin \left(\omega_{z} t\right) \frac{q V_{p-p}}{2 d_{0}^{2}} \\
& \times \sin (\omega t+\phi) z_{\max } \omega_{z} \cos \left(\omega_{z} t\right)
\end{aligned}
$$

From the trigonometric relation, $\sin \left(\omega_{z} t\right) \cos \left(\omega_{x} t\right)=\frac{1}{2}$ $\sin \left(2 \omega_{z} t\right)$, the average power absorbed during one period of on-resonance ( $\omega=2 \omega_{z}$ equals twice the trapping frequency; $\phi=\pi$ ) excitation is

$$
P=\frac{z_{\max }^{2} \omega_{z} q V_{p-p}}{4 d_{0}^{2}}
$$

By equating eq 63 with the time rate of change of ion axial energy (eq 16), we obtain

$$
P=\frac{d E_{z}}{d t}=m \omega_{z}^{2} z_{\max } \dot{z}_{\max }=\frac{z_{\max }^{2} \omega_{z} q V_{\mathrm{p}-\mathrm{p}}}{4 d_{0}^{2}}
$$

The differential equation for $\dot{z}_{\max }$

$$
\dot{z}_{\max }=\frac{q V_{\mathrm{p}-\mathrm{p}}}{2 d_{0}^{2} \omega_{z} m} z_{\max }
$$

leads to the solution,

$$
\begin{aligned}
z_{\max }(t) & =z_{\max }(0) \exp \left(\frac{q V_{\mathrm{p}-\mathrm{p}} t}{4 d_{0}^{2} \omega_{z} m}\right) \\
& =z_{\max }(0) \exp \left(\frac{V_{\mathrm{p}-\mathrm{p}} t \omega_{z}}{4 V_{\text {trap }}}\right)
\end{aligned}
$$

For in-phase on-resonance parametric excitation, the amplitude of the trapping motion increases exponentially with time (eq 66), rather than linearly with time as in standard dipolar radial excitation. The ion trapping oscillation amplitude after excitation is proportional to the initial amplitude $z_{\max }(0)$. The resonance frequency is $2 \omega_{z}$. This result is related (see below) to the (undesirable) axial ejection (" $z$ ejection") of lowmass ions during standard dipolar excitation of higher mass ions. Parametric resonant excitation at $2 \omega_{z}$ produces an electric field component along the $z$ direc-

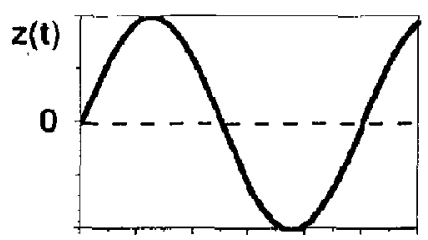

a

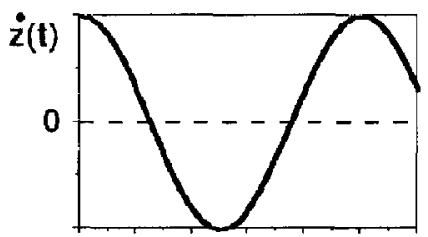

$\mathbf{b}$
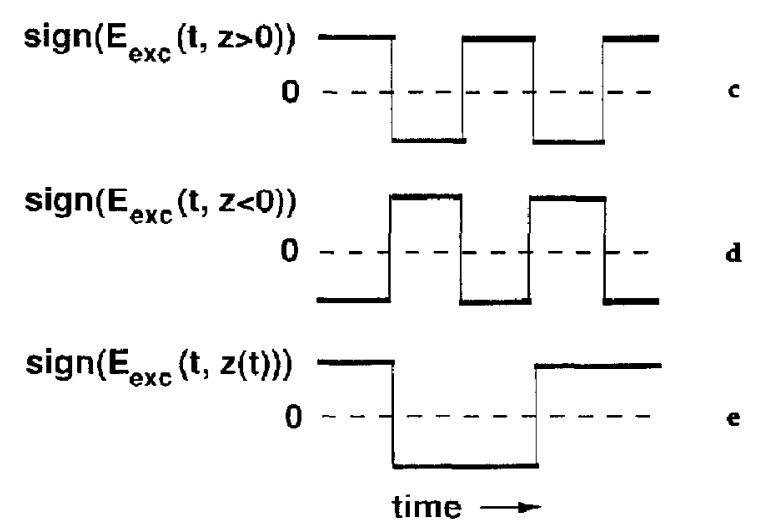

Figure 6. Time dependence of instantaneous axial position (a) and velocity (b) for ion trapping motion and sign of parametric excitation field ( $z$ component; on-resonance, in-phase) for positive $(c)$ and negative $(d)$ ion $z$ values. The sign of the electric field as seen by the ion (at position as in a) is given in (e). It is identical to the sign of the ion velocity that restrlts in a continued excitation as described in the text. 
tion, such that the sign of the electric field at the ion is the same as that of the ion $z$ velocity throughout the full period of the excitation oscillation (Figure 6). Thus, ion $z$ motion is accelerated continuously during resonant parametric excitation at $2 \omega_{z}$.

It is well known that z-ejection (intentional or unintentional) may be produced by a spatially nonuniform radial dipolar electric excitation field [8, 14, 57-65] (except on the $z$ axis, where the $z$ component of the dipolar excitation field is zero by symmetry if the trap $z$ axis is exactly aligned with the applied magnetic field). Because $z$ ejection is mass dependent (see, e.g., eq 66), it is highly undesirable because it changes the relative FT-ICR mass spectral peak area (and thus the apparent relative ion abundances); that is, it leads to mass discrimination. One source of $z$ ejection appears to result from excitation at $2 \omega_{z}$; we call it "pseudoparametric" $z$ ejection (because the geometry used is the standard radial dipolar rather than the parametric discussed above). The explanation is as follows. If an ion is not located on the $z$ symmetry axis of the trap during the excitation event, the ion experiences a $z$ component of the excitation field (unless the ion has zero trapping motion and stays strictly in the $z=0$ plane) for two possible reasons. First, a dynamic displacement from the $z$ axis is an automatic consequence of a nonzero magnetron radius. If the magnetron frequency is low, the ion may be $z$ ejected from the trap long before it has moved significantly along its magnetron trajectory. If the ion moves half a cycle in its magnetron orbit, the phase of the $z$ component of the excitation field will be reversed, leaving a zero net $z$ excitation. A nonzero magnetron radius can result from a misalignment of the electron beam for electron impact ionization or off-axis injection of externally created ions (including laser desorption/ionization methods). Second, a static ion displacement can arise from a mismatch between the trapping and/or excitation voltages applied to the electrodes of the trap. For example, if the DC potentials of two opposed side plates do not match, the effective symmetry axis of the trapping potential will be shifted from the geometric $z$ axis of the trap. An applied radial dipolar RF excitation field then exposes the ions on the shifted axis to an RF $z$ excitation field. Under the described circumstances, pseudoparametric $z$ ejection is avoided by sweeping from high to low (rather than from low to high) ion excitation frequency because the cyclotron motion is then excited first and the ions have large cyclotron radii when the excitation at $2 \omega_{z}$ is applied. Because the excitation is of standard dipolar geometry, the fast cyclotron motion (compared to the trapping motion) leads to a zero average $z$ component of the excitation field; however, one should (if possible) in addition exclude the $2 \omega_{z}$ frequencies of all ions of interest from the frequency range of the excitation, for example, by use of SWIFT $[40,41]$ excitation. This requirement may entail a careful choice of trapping voltage.
However, there is a second effective mechanism for $z$ ejection in dipolar excitation mode quite different from pseudoparametric $z$ ejection. In this second case, the $z$ ejection occurs at frequencies near the reduced cyclotron frequency $\omega_{+}$[58]. This phenomenon has recently been investigated in detail, and we refer the reader to the publication of Fujiwara et al. [66]. To perform an ICR experiment, the excitation at $\omega_{+}$cannot, of course, be avoided; however, an investigation of the response to frequency-sweep excitation again shows that a downfrequency sweep is preferred if $z$ ejection is to be avoided.

We note that both mechanisms for $z$ ejection discussed so far may be virtually eliminated by bringing the RF electric excitation field to near-perfect spatial uniformity (and no $z$ components) by addition of "guard rings" $[61,62,67]$ or by use of an "infinity" [68] or an open end-cap trap [69]. There is an interesting difference between the two $z$ ejection mechanisms that may lead to a distinction for a given experiment. In the pseudoparametric case (as for the standard parametric mode), the excitation field may be small compared to the trapping field. The electric field $z$ component is always directed toward the $z=0$ plane, but the ions nevertheless gain energy by resonant excitation during many periods of the trapping motion. In contrast, the second mechanism only works if the excitation voltage amplitude is higher than the trapping voltage. Therefore, if the excitation voltage amplitude is reduced below the trapping voltage and $z$ ejection still occurs, then it cannot be by this mechanișm.

Finally, it is worth noting that even if the dipolar excitation field has no $z$ component, ions may nevertheless be ejected axially. Ions with initially thermal velocity acquire higher radial velocity as they are excited to larger ion cyclotron radii. Ion-neutral and/or ion-ion collisions in general convert some of that increased translational energy into $z$ velocity. If the kinetic energy in the $z$ direction is sufficiently large (i.e., greater than the depth of the z-potential energy trapping well, see below), then the ion is lost from the trap. [We note that the depth of the electrostatic $z$ potential well actually increases with increasing displacement from the $z$ axis: If the trap electrodes are each held at $V_{\mathrm{T}}$, then the bottom of the $z$-potential well at the center of, e.g., a cubic trap is $V_{\mathrm{T}} / 3$, for a net well depth (difference in $z$ potential between the center of the trap and the trap electrode) of $2 V_{\mathrm{T}} / 3$; however, in the limit that an ion approaches one of the side electrodes of a cubic trap, the electric potential energy drops to zero (the potential of the side electrodes themselves), so that the well depth at the trap radius is $V_{\mathrm{T}}$, i.e., greater than the well depth along the $z$ axis. Similar arguments may be applied to other trap geometries.]

From the above discussion, it is clear that $z$ excitation is a complex problem; however, unwanted $z$ ejection may be reduced by (1) careful alignment of the 
trap with respect to ionization and/or ion injection pathway, (2) shimming of both the trapping and the RF excitation field, (3) lowering the number of ions and pressure in the trap to reduce the frequency of ion-ion and ion-neutral collisions, (4) lowering the excitation voltage amplitude below the value of the trapping voltage, (5) use of downsweep instead of upsweep in the case of frequency-sweep excitation, and (6) tailoring the excitation frequency range to include all desired cyclotron frequencies but exclude components at twice the trapping frequencies.

\section{Parametric Excitation of Radial Motion}

Next, we consider the effect of parametric excitation on the ion radial motions. The excitation geometry (Figure $1 \mathrm{~g}$ ) is identical to that discussed in the previous section. The $x$ and $y$ components of the excitation field are as in eq 61 :

$$
\begin{aligned}
& E_{x}=x \frac{V_{\mathrm{p}-\mathrm{p}}}{2 d_{0}^{2}} \sin (\omega t+\phi) \\
& E_{y}=y \frac{V_{\mathrm{p}-\mathrm{p}}}{2 d_{0}^{2}} \sin (\omega t+\phi)
\end{aligned}
$$

The power (averaged over one cycle of the excitation frequency) absorbed into the radial motions (eq 20, eq 67) is

$$
P(t)=\frac{q V_{\mathrm{p}-\mathrm{p}}}{2 d_{\mathrm{o}}^{2}} \sin (\omega t+\phi)[x \dot{x}+y \dot{y}]
$$

Substituting for $x, y, \dot{x}$, and $\dot{y}$ from eq $2 \mathrm{a}-\mathrm{h}$, we find that

$$
\begin{aligned}
x \dot{x}+y \dot{y}= & \omega_{p} \rho_{+} \rho_{-}\left[\sin \left(\omega_{-} t\right) \cos \left(\omega_{+} t\right)\right. \\
& \left.-\cos \left(\omega_{-} t\right) \sin \left(\omega_{+} t\right)\right] \\
= & -\omega_{p} \rho_{+} \rho_{-} \sin \left(\omega_{p} t\right)
\end{aligned}
$$

so that

$$
P(t)=-\omega_{\mathrm{p}} \rho_{+} \rho_{-} \frac{q V_{\mathrm{p}-\mathrm{p}}}{2 d_{0}^{2}} \sin (\omega t+\phi) \sin \left(\omega_{\mathrm{p}} t\right)
$$

Again, we are interested in the in-phase on-resonance case $\phi=\pi, \omega=\omega_{p}$, for which eq 70 simplifies to

$$
P=\omega_{\mathrm{p}} \rho_{+} \rho_{-} \frac{q V_{\mathrm{p}-\mathrm{p}}}{4 d_{0}^{2}}
$$

In contrast to the standard dipolar radial excitation the resonance frequency, $\omega_{p}$, is a combination of the frequencies of two ion motional modes: $\omega_{p}=\omega_{+}-\omega_{-}$. If an energy quantum from the excitation field is absorbed, the radial energy, $\mathrm{E}_{+}+\mathrm{E}_{-}$changes from $\left(\mathrm{n}_{+}+1 / 2\right)(\mathrm{h} / 2 \pi) \omega_{+}-\left(n_{-}+1 / 2\right)(\mathrm{h} / 2 \pi) \omega_{-}$to $\left(\mathrm{n}_{+}+\right.$ $3 / 2)(h / 2 \pi) \omega_{+}-\left(n_{-}+3 / 2\right)(h / 2 \pi) \omega_{-}$. Thus, the rates of change of the cyclotron and the magnetron energies are related by

$$
\frac{d E_{+}}{d t}=-\frac{\omega_{+}}{\omega_{-}} \frac{d E}{d t}
$$

Therefore, the power absorbed into both the magnetron and cyclotron orbital motions may be expressed by the change in the magnetron or cyclotron orbital energy only:

$$
\begin{aligned}
& P=\frac{d\left(E_{+}+E_{-}\right)}{d t}=\frac{\omega_{p}}{\omega_{+}} \frac{d E_{+}}{d t} \\
& P=\frac{d\left(E_{+}+E_{-}\right)}{d t}=-\frac{\omega_{p}}{\omega_{-}} \frac{d L_{-}}{d t}
\end{aligned}
$$

Moreover, from eqs 14,15 , and 72 , the time rates of change in magnetron and cyclotron radii are related by

$$
\rho_{+} \frac{d \rho_{+}}{d t}=\rho_{-} \frac{d \rho_{-}}{d t}
$$

By expressing the total power absorption in terms of changes in the magnetron and cyclotron orbital radii (combine eqs 14, 15, and 73),

$$
\begin{aligned}
& P=m \omega_{\mathrm{p}}^{2} \rho_{+} \frac{d \rho_{+}}{d t} \\
& P=m \omega_{\mathrm{p}}^{2} \rho_{-} \frac{d \rho_{-}}{d t}
\end{aligned}
$$

we arrive (combine eq 71 with eq $75 a$ or b) at a system of two coupled differential equations for $\rho_{+}$and $\rho_{-}$:

$$
\begin{aligned}
& \dot{\rho}_{+}-\rho_{-} \frac{q V_{\mathrm{p}-\mathrm{p}}}{4 m \omega_{\mathrm{p}} d_{0}^{2}}=0 \\
& \dot{\rho}_{-}-\rho_{+} \frac{q V_{\mathrm{p}-\mathrm{p}}}{4 m \omega_{\mathrm{p}} d_{0}^{2}}=0
\end{aligned}
$$

This system of differential equations is readily solved by standard methods, such as the Laplace transform. For an initial cyclotron orbital radius $\rho+(0)$ and ion magnetron radius $\rho_{-}(0)$,

$$
\begin{aligned}
& \rho_{+}(t)=\rho_{+}(0) \cosh (\gamma t)+\rho_{-}(0) \sinh (\gamma t) \\
& \rho_{-}(t)=\rho_{+}(0) \sinh (\gamma t)+\rho_{-}(0) \cosh (\gamma t)
\end{aligned}
$$

with

$$
\gamma=\frac{q V_{\mathrm{p}} \mathbf{p}}{4 m \omega_{\mathrm{p}} d_{0}^{2}}
$$

For the usual case that $\omega_{-} \ll \omega_{+}$, we may approximate $\omega_{\mathrm{p}}=\omega_{+}-\omega_{-}=\omega_{c}-2 \omega_{-} \approx \omega_{\mathrm{c}}$, so that

$$
\gamma \approx \frac{V_{\mathrm{p}-\mathrm{p}}}{4 B d_{0}^{2}}
$$


For parametric radial excitation, both the cyclotron and magnetron radii increase exponentially. In all parametric excitation experiments performed to date, the initial cyclotron orbital radius of the ions was negligibly small. In that case, eq 77 reduce to

$$
\begin{aligned}
& \rho_{+}(t)=\rho_{-}(0) \sinh (\gamma t) \\
& \rho_{-}(t)=\rho_{-}(0) \cosh (\gamma t)
\end{aligned}
$$

(see Figure 5). Although the asymptotic behavior $(t \gg$ $1 / \gamma)$ of magnetron and cyclotron orbital radii is the same, the initial increase takes place almost entirely in the cyclotron orbital motion (see Figure 7). To maximize the detected ion signal, an ion trap operating in parametric mode should be designed to allow for the largest possible radial distance variation, namely, from $\left|\rho_{+}-\rho_{-}\right|$to $\left(\rho_{+} \mid \rho_{-}\right)$[33]. We therefore now consider the optimal radial displacement from the trap $z$ axis at which the ions should be introduced initially in a trap operated with parametric mode excitation/detection. The largest detected signal is expected if, after excitation of duration $T$, the magnetron and cyclotron orbital radii are equal and their sum is slightly smaller than the trap radius $p_{0}$. Therefore,

$$
\begin{aligned}
& \rho_{+}(T)=\rho_{0} / 2=\rho_{-}(0) \sinh (\gamma T) \\
& \rho_{-}(T)=\rho_{0} / 2=\rho_{-}(0) \cosh (\gamma T)
\end{aligned}
$$

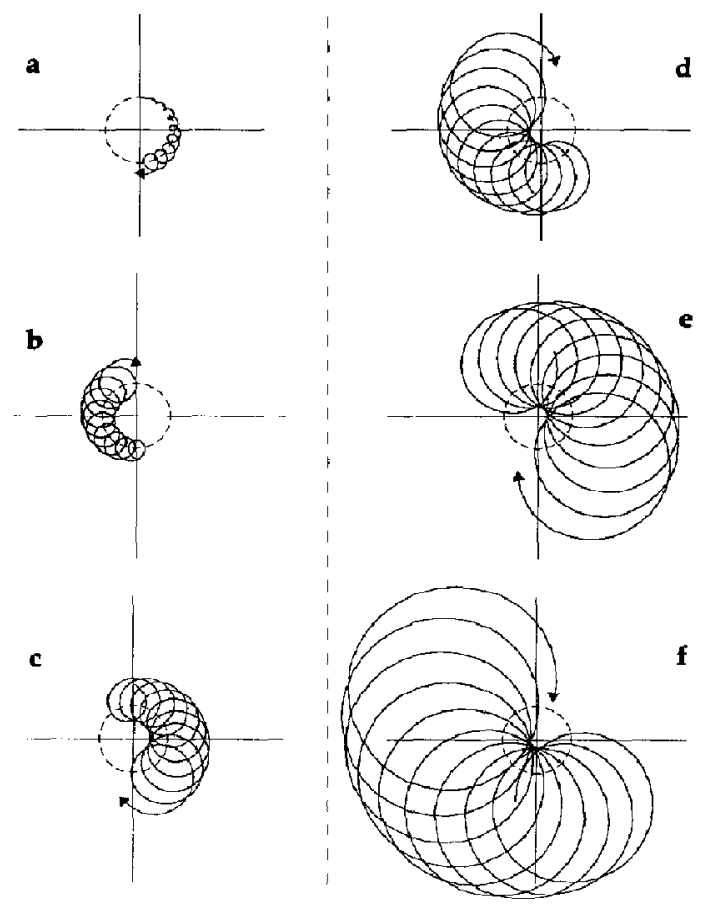

Figure 7. Radial ion motions for parametric excitation (see eq 77). $\omega_{+} / \omega_{-}=20$. Initial magnetron orbit circle is shown as a dashed line. Note that both the magnetron and ion cyclotron orbital radii increase with time. implying that $\sinh (\gamma T)=\cosh (\gamma T)$; however, there is no point of intersection for the hyperbolic sine and cosine functions, although they approach each other asymptotically as the argument, $\gamma T$ increases. Thus, eq 80 require that one choose a relatively small initial magnetron radius $\rho_{-}(U)$. Unfortunately, space-charge repulsion limits the number of ions that may be packed close to the central $z$ axis of the trap. Also, very small initial radii are not desirable because the excitation is essentially exponential, and a small deviation from the initial position (when close to the trap center) translates into a very large deviation from the intended cyclotron radius after excitation. Thus, for the traditional Penning trap with two endcaps and a ring electrode (as well as for traditional cubic or cylindrical traps), optimization of the initial magnetron radius poses a problem.

In the two-electrode hyperbolic trap with concentric core and ring electrodes $[12,21]$, the ion optimal initial position is easily calculated as follows. Let $\rho_{\text {core }}$ and $\rho_{\text {ring }}$ be the minimal (waist) radii for the core and ring electrodes, respectively. The maximal detected signal results from a magnetron radius (after excitation) exactly half-way between the electrodes and a cyclotron radius (again after excitation) of half the distance between the electrodes:

$$
\begin{aligned}
& \rho_{-}(T)=\left(\rho_{\text {ring }}+\rho_{\text {core }}\right) / 2 \\
& \rho_{+}(T)=\left(\rho_{\text {ring }}-\rho_{\text {core }}\right) / 2
\end{aligned}
$$

From eq 81, the optimal initial magnetron radius $\rho_{-}(0)$ can be determined to be the geometric average of the core and ring electrodes [21],

$$
\rho_{-}(0)=\sqrt{\rho_{\text {ring }} \rho_{\text {care }}}
$$

and the corresponding excitation duration $T$ is

$$
T+\frac{1}{2 \gamma} \log _{e}\left(\frac{\rho_{\text {ring }}}{\rho_{\text {core }}}\right)
$$

The optimal initial magnetron radius may also be expressed in terms of the final magnetron and cyclotron radii:

$$
\rho_{-}(0)=\sqrt{\rho_{-}^{2}(T)-\rho_{+}^{2}(T)}
$$

(Of course, the implementation of eqs 82 and 84 in practice will be complicated by the distribution in trapping oscillation energy of the ions.) A more comprehensive discussion and experimental results of the performance of this new trap design have recently been presented [21]. 


\section{Azimuthal Quadrupolar Excitation of Radial Motion}

In the preceding section, we dealt with an axial quadrupolar excitation. Here we consider the quadrupolar excitation field of radial (azimuthal) geometry (Figure 1h), which can be achieved in a cubic trap by connecting the excitation signal to one pair of opposed side plates and the same signal phase-shifted by $\pi$ radians to the other pair of opposed side plates. To a first approximation, the excitation RF electric field can be written

$$
\begin{aligned}
& E_{x}=+x C \sin (\omega t+\phi) \\
& E_{y}=-y C \sin (\omega t+\phi)
\end{aligned}
$$

For the cubic trap, the constant $C$ follows from essentially the same calculation as for the trapping potential [4] (due to the symmetry of the cubic trap),

$$
C=\frac{3 \alpha V_{\mathrm{p}-\mathrm{p}}}{2 a^{2}}
$$

The instantaneous power absorption for the radial motions (with $x, y, \dot{x}$, and $\dot{y}$ from eqs 1 and 2) amounts to

$$
\begin{aligned}
P(t)= & q C \sin (\omega t+\phi)\left[\rho_{1}^{2} \omega_{1} \sin \left(2 \omega_{+} t\right)\right. \\
& \left.+\rho_{-}^{2} \omega_{-} \sin \left(2 \omega_{-} t\right)+\rho_{+} \rho_{-} \omega_{\mathrm{c}} \sin \left(\omega_{\mathrm{c}} t\right)\right]
\end{aligned}
$$

There are clearly three excitation frequencies that produce a long-term (average) effect on the ion motion. Here excitation at frequencies, $2 \omega_{+}$and $\omega_{c r}$ is discussed. (The line of reasoning for excitation at $2 \omega_{-}$is analogous to that for $2 \omega$. .)

Excitation at $2 \omega_{+}$. Again we are interested in the in-phase on-resonance case $\phi=0, \omega=2 \omega_{+}$, for which the power absorption averaged over one cycle of the excitation frequency is

$$
P=\frac{q C \rho_{+}^{2} \omega_{+}}{2}
$$

As above (see eq 28 on radial dipolar excitation), we set $P$ equal to the time rate of change of cyclotron orbital energy,

$$
P=\frac{d E_{1}}{d t}=m \omega_{\mathrm{p}} \omega_{+} \rho_{+} \dot{\rho}_{+}
$$

to yield a differential equation for ion cyclotron orbital radius $\rho_{+}$:

$$
\dot{\rho}_{+}=\frac{q C}{2 m \omega_{\mathrm{p}}} \rho_{+}
$$

Equation 90 may be solved to yield

$$
\rho_{+}(t)=\rho_{+}(0) \exp \left(\frac{q C}{2 m \omega_{\mathrm{p}}} t\right)
$$

Equation 91 shows that the ion cyclotron orbital radius increases exponentially from its initial value. The exponent is inversely proportional to $\left(\omega_{\mathrm{p}} m / q\right)$. Because $\omega_{\mathrm{p}} \approx \omega_{\mathrm{c}}=q B / m$, there is (to first order) no dependence of excited ion cyclotron orbital radius on ion mass-to-charge ratio.

Excitation at $\omega_{c}$. On application of azimuthal quadrupolar RF excitation at $\omega_{\mathrm{c}}=\omega_{+}+\omega_{-}$, both the magnetron and cyclotron orbital motions are affected (as was the case for parametric excitation at the combination frequency $\omega_{\mathrm{p}}=\omega_{+}-\omega_{-}$, as discussed above). On absorption of an energy quantum from the excitation field, the total radial energy $E_{+}+E_{-}$changes from $\left(n_{+}+1 / 2\right)(h / 2 \pi) \omega_{+}-\left(n_{-}+1 / 2\right)(h / 2 \pi) \omega_{-}$to $\left(n_{+}+3 / 2\right)(h / 2 \pi) \quad \omega_{+}-\left(n_{-}-1 / 2\right)(h / 2 \pi) \quad \omega_{-}$. Similarly, for (stimulated) emission of an energy quantum, the total radial energy changes from $\left(n_{+}+\right.$ $1 / 2)(h / 2 \pi) \omega_{+}-\left(n_{-}+1 / 2\right)(h / 2 \pi) \omega_{-}$to $\left(n_{+}-\right.$ $1 / 2)(h / 2 \pi) \omega_{+}-\left(n_{-}+3 / 2\right)(h / 2 \pi) \omega_{-}$. In either case, the time rates of change of the magnetron and cyclotron orbital energies are related by

$$
\frac{d E_{+}}{d t}=\frac{\omega_{+}}{\omega_{-}} \frac{d E_{-}}{d t}
$$

and the change of magnetron and cyclotron radii are related by

$$
\rho_{+} \frac{d \rho_{+}}{d t}=-\rho-\frac{d \rho_{-}}{d t}
$$

From the change in total energy expressed in terms of change in the cyclotron or magnetron energy alone (see Parametric Excitation of Radial Motion),

$$
\begin{aligned}
\frac{d E_{\text {total }}}{d t} & =\frac{d E_{+}}{d t}+\frac{d E_{-}}{d t} \\
& =\frac{\omega_{\mathrm{c}}}{\omega_{-}} \frac{d E_{-}}{d t}=\frac{\omega_{\mathrm{c}}}{\omega_{+}} \frac{d E_{+}}{d t}
\end{aligned}
$$


one arrives at the following system of coupled differential equations:

$$
\begin{aligned}
& \dot{\rho}_{+}=\frac{q C}{2 m \omega_{p}} \rho_{-} \\
& \dot{\rho}_{-}=-\frac{q C}{2 m \omega_{p}} \rho_{+}
\end{aligned}
$$

As it happens, this system of differential equations is the same as the well-studied Hamiltonian equations governing the position and momentum of a harmonic oscillator, as explained in any good introductory classical mechanics text [70]. The solutions are

$$
\begin{aligned}
& \rho_{+}(t)=\rho_{-}(0) \sin \left(\omega_{\mathrm{b}} t\right)+\rho_{+}(0) \cos \left(\omega_{\mathrm{b}} t\right) \\
& \rho_{-}(t)=\rho_{-}(0) \cos \left(\omega_{\mathrm{b}} t\right)-\rho_{+}(0) \sin \left(\omega_{\mathrm{b}} t\right)
\end{aligned}
$$

in which $a_{\mathrm{b}}$ denotes the "beat" frequency,

$$
\omega_{\mathrm{b}}=\frac{q C}{2 m \omega_{\mathrm{p}}}
$$

between the cyclotron and the magnetron motions (Figure 8). If the ions start out in just one mode, for example, a pure magnetron motion with $p_{+}(t=0)=0$, azimuthal quadrupolar excitation progressively reduces the magnetron radius and increases the $c y-$ clotron orbital radius until pure cyclotron orbital motion is attained at time $\left(\pi / 2 \omega_{\mathrm{b}}\right)$. Following another period $\left(\pi / 2 \omega_{b}\right)$, the ion trajectory converts back to a pure magnetron mode; however, after time $\left(\pi / \omega_{b}\right)$, there is a phase shift of $\pi$ with respect to what the magnetron motion would have had in the absence of azimuthal quadrupolar excitation. This phase shift is evidenced in the above sulution by a negative value of the instantaneous magnetron radius. In general, the initial magnetron and cyclotron radii $\rho_{+}(0)$ and $\rho_{-}(0)$, du nut fully determine the trajectory of the ion. Depending on the phase of the excitation signal, either the magnetron radius or the cyclotron orbital radius increases while the other decreases. At any rate, an admixture of both motions can be converted into one pure mode by this method, making it a means of axialization of initially off-axis ions. Azimuthal quadrupolar excitation has been used for high-precision (non-FT) ICR/MS [22, 71] of short-lived isotopes as well as for cooling ions in the presence of a buffer gas [24]. The latter scheme has recently been adapted for FT-ICR/MS [25]. The interconversion between cyclotron and orbital modes under the influence of
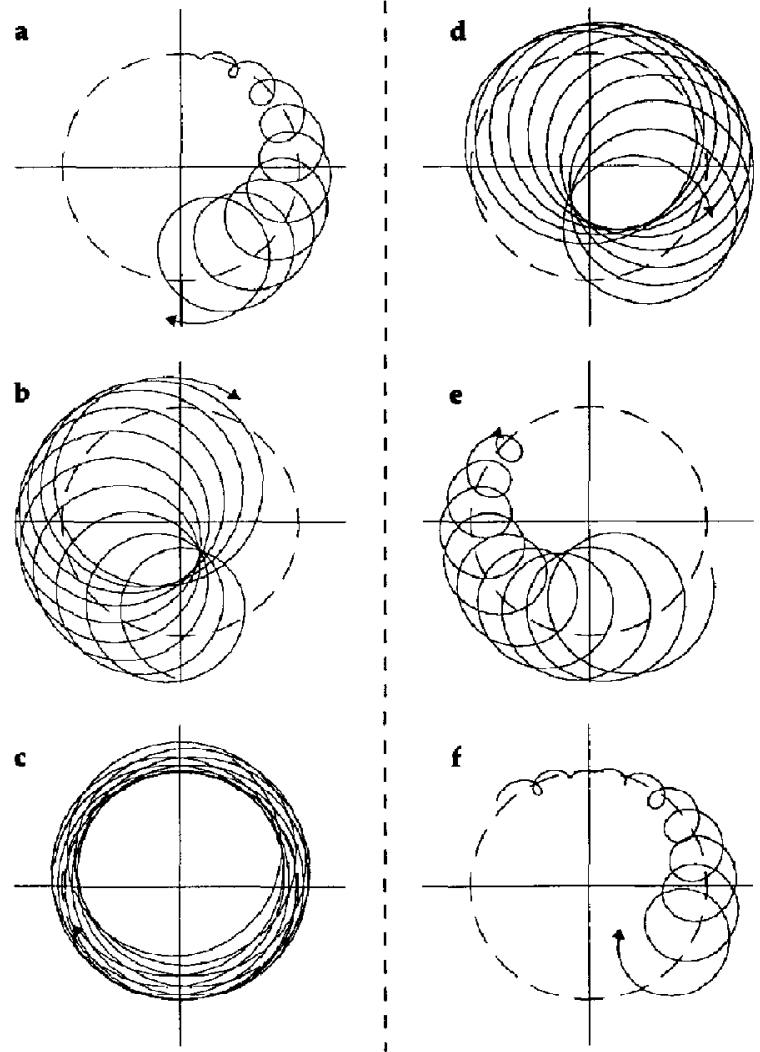

Figure 8. Radial ion motions for quadrupolar excitation according to the analytical eq 96 (see similar plots $[22,23]$, in which the trajectories were evaluated by mumerical integration of the equations of motion): $\omega_{+} / v \omega_{-}=20$. Dashed circles as in Figure 5. Note that an in that begins with pure magnetron motion $\left(\rho_{+}=0\right.$ at time zero at upper left in diagram) evolves to pure cyclotron motion $\left(\rho_{-}=0\right.$ at bottom left $)$ and back to pure magnetron motion (bottom right). Such excitation is useful for axialization of initially off-axis ions (see text).

quadrupolar excitation in ICR is formally analogous to the evolution of a two-state spin system governed by Bloch equations; in fact, the analogy extends to $180^{\circ}$ pulse excitation and adiabatic rapid passage [72].

Very recently, Guan et al. [26] have pointed out for FT-ICR/MS that quadrupolar excitation of $x z$ symmetry (as opposed to the just-discussed quadrupolar excitation of $x y$ symmetry) couples axial ion motion to the magnetron and cyclotron motions, as previously noted for single-frequency ICR [73-75]. Ions may be resonantly excited at any of the frequencies $\omega_{z}+\omega_{-}, \omega_{z}-$ $\omega_{-}, \omega_{z}+\omega_{+}$, and $\omega_{+}-\omega_{z}$. For example, excitation at $\omega_{z}+\omega_{-}$effects a periodic interconversion between axial and magnetron motion, similar to the magnetronto-cyclotron interconversion described by eq 96. Although the simple cubic trap shown in Figure 1a must be modified by further segmentation of the side plates, the quadrupolar $x z$ excitation experiment is otherwise 
quite analogous to the quadrupolar $x y$ excitation described above and is not treated further here.

\section{Limitations of the Present Method}

In this work, we have assumed that each ion motional mode (or combination mode) maintains a constant frequency during the excitation process. That assumption is violated in at least three circumstances. First, for a nonquadrupolar electrostatic trapping potential (e.g., nonhyperbolic trap and/or space-charge potential), the ion cyclotron orbital frequency can vary with cyclotron orbital radius; that shift can be minimized by reduction in trap potential and/or reduction in number of trapped ions. Second, for ions of low mass-to-charge ratio, the relativistic increase in ion mass with increasing ion speed translates into a lowering of ion $\mathrm{cy}^{-}$ clotron orbital frequency with increasing ion cyclotron orbital radius; that shift can be calculated for a given excitation geometry and known excitation magnitude and duration, and is in any case usually negligible for ions of chemical interest (say, $\geq 20 \mathrm{w}$ /elementary charge). Third, in standard dipolar radial excitation, for example, the instantaneous ion cyclotron orbital frequency $d \phi / d t$ of an ion with nonzero initial cyclotron orbital radius initially increases (ion accelerates in its orbit) or decelerates (ion decelerates in its orbit), depending on the initial ion cyclotron orbital phase, during the first few excitation cycles [36] until a steady-state $\phi=\pi / 2$ phase relationship between cxcitation and ion motion is establishcd; the ICR or bital radius then expands linearly according to eq 30 . Alternatively, one may consider that the ICR frequency remains constant, whereas the relative proportion of in-phase and $90^{\circ}$ out-phase components varies with time for a few motional cycles until a steady-state phase difference of $\phi=\pi / 2$ radians is reached between excitation and ion motion. In any case, the on-resonant condition applies as soon as the motion of the ion packet becomes coherent [i.e., $\rho_{+}(t) \gg \rho_{+}(0)$ ].

We have considered here the power absorption averaged over one cycle of the excitation frequency; however, tor standard dipolar (or quadrature) radial excitation, for example, we previously noted that the instantaneous center of the magnetron orbit oscillates (or rotates) during excitation; that oscillation (or rotation) may be reduced by lowering the excitation RF electric field amplitude [36]. We also assumed that the change per cycle of, for example, the ion cyclotron orbital radius is small compared to the orbital radius itself; that assumption is clearly violated for radial quadrupolar excitation at $\omega_{c}$, for which the orbital radius periodically evolves through a radius of zero. A rigorous discussion of averaging methods for the description of FT-ICR ion motions has recently been presented [34]. Perturbation methods applied to Penning traps are discussed in detail by Kretzschmar $[76,77]$.

\section{Conclusions and Future Outlook}

In this work, we have tried to provide a systematic and consistent introduction to the family of excitation geometries for single-frequency resonant, in-phase excitation in FI-ICR mass spectrometry. The effects of standard dipolar radial, quadrature, feedback, axial dipolar, parametric, and quadrupolar excitation as well as velocity-proportional damping on the ion motion have been discussed. Table 1 lists analytical expressions for the amplitudes of ion motion as a function of excitation frequency, excitation period, excitation amplitude, trapping potential, trap shape and size, and ion mass-to-charge ratio. Figure 9 shows graphically how the amplitude of ion motion varies during the excitation period for each of several types of resonant excitation modes. Excitation amplitude varies with time linearly (dipolar radial, dipolar axial, or quadrature radial excitation), exponentially (frictional damping or feedback, parametric axial, or azimuthal quadrupolar excitation), hyperbolic cosine and sine (parametric radial excitation), and cosine and sine (azimuthal quadrupolar excitation).

The excitation configurations discussed here include the principal excitation modes for a typical six-electrode (e.g., cubic) trap. Other excitation modes can be generated as higher order terms of an appropriate series expansion. For example, as noted above, the radial and trapping motions can be coupled by higher order terms of the standard dipolar radial excitation geometry in a cubic trap (e.g., at the frequencies $\omega_{+} \pm$ $2 \omega_{z}$ [78]; however, a modified trap geometry, such as segmentation of the electrodes, is much more efficient in exciting multiple harmonic and/or combination frequencies. The possibility of coupling between axial and radial motions has been mentioned earlier (Azimuthal Quadrupolar Excitation of Radial Motion). The present treatment could also be extended to higher order terms of multipolar (e.g., octupolar) fields. Note that the concept of feedback excitation introduced above for the special case of dipolar radial excitation can be extended to other excitation modes as well.

Finally, we point out that the excitation frequency suitable for any of the above-listed excitation geometries is the same as the ion signal frequency for detection with the same configuration, provided that the appropriate ion motions have been excited, as previously discussed for the particular case of radial quadrupolar detection [33]. This result follows from the reciprocity theorem for image charges $[6,29,30]$, which states that the ratio of the image charge induced on one or more electrodes of the trap to the charge of the ion is the same as the ratio of the change in potential at a given ion position to the applied potential change on the same electrode(s). That applied potential (more precisely, its gradient) drives the ion motion during excitation. The image charge from that coherently moving ion packet produces the FT-ICR time-domain signal. Thus, by extending the reciprocity 
Table 1. Amplitude of ion motional modes in response to harmonic resonant single-frequency excitation for several excitation configurations

\begin{tabular}{|c|c|c|c|}
\hline $\begin{array}{l}\text { Excitation } \\
\text { mode }\end{array}$ & $\begin{array}{l}\text { Resonance } \\
\text { frequency }\end{array}$ & Equation & Number \\
\hline Dipolar radial & $\omega_{+}$ & $p_{+}(t)-\frac{q \beta V_{p-p} t}{2 m a \omega_{p}}$ & $(3 \mathrm{Oa})$ \\
\hline Dipolar radia ${ }^{\star}$ & $\omega_{\mathrm{c}}$ & $\rho_{\mathrm{c}}(t)=\frac{q \beta V_{\mathrm{p}-\mathrm{p}} t}{2 m a \omega_{\mathrm{c}}}$ & (3Ob) \\
\hline Dipolar radial & $\omega_{-}$ & $\rho_{-}(t)=\rho_{-}(0)-\frac{q \beta V_{p-p} t}{2 m a \omega_{p}}$ & (35) \\
\hline Quadrature radial & $\omega_{+}$ & $\rho_{+}(t)=\frac{q \beta V_{\mathrm{p}-\mathrm{p}} t}{m \theta \omega_{\mathrm{p}}}$ & $(40 a)$ \\
\hline Quadrature radial ${ }^{\mathbf{a}}$ & $\omega_{\mathrm{c}}$ & $\rho_{t}(t)=\frac{q \beta V_{p-p} t}{m a \omega_{k}}$ & $(40 b)$ \\
\hline Feedback radial & $\omega_{1}$ & $\rho_{+}(z)=\rho_{1}(O) \exp \left(\frac{q A}{2 m \omega_{p}} t\right)$ & (45) \\
\hline \multirow[t]{3}{*}{ Frictional damping } & - & $\rho_{+}(t)=\rho_{+}(0) \exp \left(-\frac{f \omega_{+}}{m \omega_{p}} t\right)$ & $(51 \mathrm{a})$ \\
\hline & $\ldots$ & $\rho_{-}(t)=\rho_{-}(0) \exp \left(+\frac{f \omega_{-}}{m \omega_{p}} t\right)$ & $(51 b)$ \\
\hline & - & $z_{\max }(t)=z_{\max }(0) \exp \left(-\frac{t}{2 m} t\right)$ & $(51 \mathrm{c})$ \\
\hline Dipole axial & $\omega_{z}$ & $z_{\max }(t)=\frac{q E_{0} t}{2 m \omega_{z}}$ & (57) \\
\hline Dipole axial & $\omega_{\mathrm{z}}$ & $z_{\max }(t)=\frac{\beta V_{p-p} t}{2 a} \sqrt{\frac{q}{m V_{\text {trap }}} \frac{a^{2}}{2 \alpha}}$ & (60) \\
\hline Parametric axial & $2 \omega_{2}$ & $z_{\text {max }}(t)=z_{\text {max }}(0) \exp \left(\frac{V_{p-p} t w_{c}}{4 V_{\text {trap }}}\right)$ & $(66)$ \\
\hline \multirow[t]{2}{*}{ Parametric radial } & $\omega_{\mathrm{p}}$ & $\begin{array}{l}\rho_{+}(t)=\rho_{+}(0) \cosh (\gamma t)+\rho_{-}(0) \sinh (\gamma t) \\
\rho_{-}(t)=\rho_{+}(0) \sinh (\gamma t)+\rho_{-}(0) \cosh (\gamma t)\end{array}$ & $\begin{array}{l}(77 a) \\
(77 b)\end{array}$ \\
\hline & & in which $\gamma=\frac{q V_{p-p}}{4 m \omega_{p} d_{0}^{2}}$ & $(77 c)$ \\
\hline Azimuthal quadrupolar & $2 \omega_{+}$ & $\rho_{+}(t)=\rho_{+}(0) \exp \left(\frac{q C}{2 m \omega_{\mathrm{p}}} t\right)$ & $(97)$ \\
\hline Azimuthal quadrupolar & $\omega_{\mathrm{c}}$ & $\begin{array}{l}\rho_{+}(t)=\rho_{-}(0) \sin \left(\omega_{\mathrm{b}} t\right)+\rho_{+}(0) \cos \left(\omega_{\mathrm{b}} t\right) \\
\rho_{--}(t)=\rho_{-}(0) \cos \left(\omega_{\mathrm{b}} t\right)-\rho_{+}(0) \sin \left(\omega_{\mathrm{b}} t\right) \\
\text { in which } \omega_{\mathrm{b}}=\frac{q C}{2 m \omega_{\mathrm{p}}}\end{array}$ & $\begin{array}{l}(96 a) \\
(96 b) \\
(96 c)\end{array}$ \\
\hline
\end{tabular}

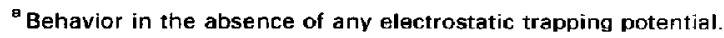

relation to the successive positions of an ion as it proceeds along its motional trajectory, we see that detected and excitation frequencies are the same if the same electrode configuration is used for both excitation and detection.

Despite the above-listed limitations, analysis at the present level of approximation has already led to several interesting new results, such as the optimization of ion initial position for parametric excitation [21] and the axialization of ions by use of quadrupolar excita- tion [23-25]. The model also accounts for frictional damping of heavy ions, leading to ion "remeasurement" [55]. The "feedback" excitation scheme may be attractive for excitation of light ions of relativistically shifted ion cyclotron orbital frequency [53].

\section{Acknowledgments}

This work was supported by the National Science Foundation. (CHE-90-21058) and The Ohio State University. L.S. acknowl- 
a

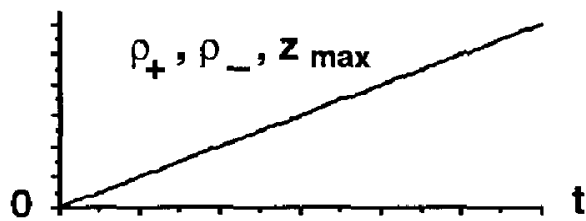

b

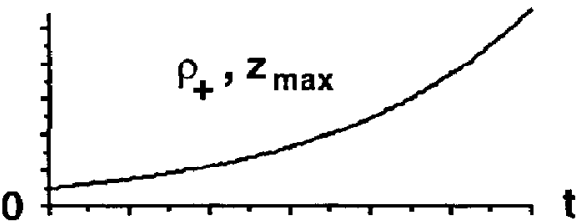

c

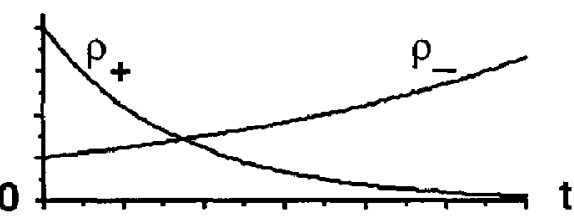

d

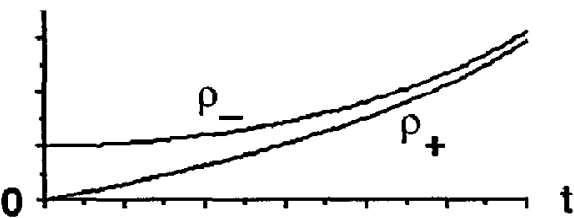

$\mathbf{e}$

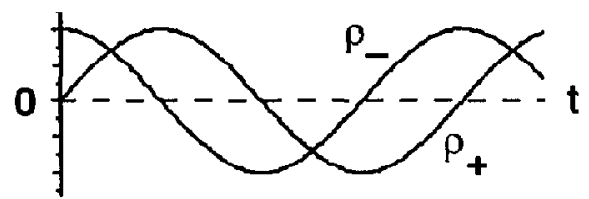

Figure 9. Ion motional amplitude versus time during various excitation modes: (a) linear, for dipolar radial (at $\omega_{+}$and $\omega_{-}$, eqs 30 and 35), and dipolar axial (at $\omega_{z}$, eqs 57 and 60), and quadrature radial (at $\omega_{+}$and $\omega_{-}$, eq 40) excitation; (b) exponential for feedback (at $\omega_{+}$, eq 45), parametric axial (at $2 \omega_{z}$, eq 66), and azimuthal quadrupolar (at $2 \omega_{+}$, eq 91) excitation; (c) frictional damping (at $\omega_{+}$and $\omega_{-}$, eq 51); (d) hyperbolic cosine and sine for parametric tadial excitation [at $\omega_{p}$, eq 77 with $p_{+}(t=0)$ $=0]$; and (e) cosine and sine for azimuthal quadrupolar excitation [at $\omega_{\mathrm{c}}$ eq 96 with $\rho_{+}(t=0)=0$ ); negative radius corresponds to $180^{\circ}$ phase shift.

edges an Ohio State University Pustdoctoral Fellowship and a stipend from the Deutsche Forschungsgemeinschaft.

\section{References}

1. Marshall, A. G.; Grosshans, P. B. Anal. Chem. 1991, 63, 215A-229A.

2. Marshall, A. G.; Schweikhard, L. Int. J. Mass Spectrom. Ion Proc. 1992, $118,119,37-70$.

3. Grosshans, P. B.; Marshall, A. G. Int. J. Mass Spectrom. Ion Proc. 1990, 100, 347-379.

4. Grosshans, P. B.; Marshall, A. G. Anal. Chem. 1991, 63, 2057-2061.

5. Comisarow, M. B. Int. I. Mass Spectrom. Ion Phys. 1981, 37, 251-257.
6. Grosshans, P. B.; Shields, P. J.; Marshall, A. G. J. Chem. Phys. 1991, 94, 5341-5352.

7. Lee, S. H.; Wanczek, K.-P; Hartmann, H. Adv. Mass Spectrom. 1980, 8B, 1645-1647.

8. Kofel, P.; Allemann, M.; Kellerhals, H.; Wanczek, K.-P. Int. J. Mass Spectrom. Ion Proceedings 1986, 74, 1-12.

9. Wang, M.; Ledford, E. B. Jr.; Marshall, A. G. Presented at FACSS XIV; Detroit, MI, 1987; abstract 43.

10. Schweikhard, L.; Blundschling, M.; Jertz, R.; Kluge, H.-J. Reo. Sci. Instrum. 1989, 60, 2631.

11. Schweikhard, L; Blundschling, $\mathbf{M}_{\text {; }}$ Jertz, $\mathbf{R}_{\text {; }}$ Kluge, H.-J. Int. I. Mass Spectrom. Ion Proceedings 1989, 89, R7-R12.

12. Schweikhard, L.; Lindinger, M.; Kluge, H.-J. Rev. Sci. Instrum. $1990,61,1055-1058$.

13. Schweikhard, L.; Lindinger, $M_{\text {; }}$ Kluge, H.-J. Int. J. Mass Spectrom. Ion Proc. 1990, 98, 2533.

14. Yin, W. W.; Wang, M.; Marshall, A. G.; Ledford, E. B. Jr. J. Amer. Soc. Mass Spectrom. 1992, 3, 188-197.

15. Comisarow, M. B.; Marshall, A. G. Chem. Phys. Left. 1974, 25, $282-283$.

16. Guan, S.; Gorshkov, M. V.; Marshall, A. G. Chem. Phys. Lett. $1992,198,143-148$.

17. Marshall, A. G.; Verdun, F. R. Fourier Transforms in NMR, Optical, and Mass Spectrometry: A User's Handbook; Elsevier: Amsterdam, 1990.

18. Hunter, R. L.; Sherman, M. G.; McIver, R. T. Jr. Int. J. Mass Spectrom. Ion Phys. 1983, 50, 259-274.

19. Beauchamp, J. L.; Armstrong, J. T. Rev. Sci. Instrum. 1969, 40, $123-128$

20. Rempel, D. L.; Ledford, E. B. Jr.; Huang, S. K.; Gross, M. L. Anal. Chem. 1987, 59, 2527-2532.

21. Marto, J. A.; Schweikhard, L.; Marshall, A. G. Proceedings of the 40th ASMS Conference on Mass Spectrometry and Allied Topics; Washington, DC, May 31-June 5, 1992; pp 1729-1730.

22. Becker, S.; Bollen, G.; Kern, F.; Kluge, H.-J.; Moore, R. B.; Savard, G.; Stolzenberg, H.; Collaboration, I. Int. I. Mass Spectrom. Ion Proceedings 1990, 99, 53-77.

23. Bollen, G.; Moore, R. B.; Savard, G.; Stolzenberg, H. Appl. Phys. 1990, 68, 4355-4374.

24. Savard, G.; Becker, S.; Bollen, G.; Kluge, H.-J.; Moore, R. B.; Schweikhard, L.; Stolzenberg, H.; Wiess, U. Phys. Lett. A 1991, $158,247-252$.

25. Schweikhard, L.; Guan, S.; Marshall, A. G. Int. J. Mass Spectrom. Ion Proceedings 1992, 120, 71-83.

26. Guan, S.; Xiang, X.; Marshall, A. G. Int J. Mass Spectrom Ian Proceedings 1993.

27. Brown, L. S.; Gabrielse, G. Rev. Mod. Phys. 1986, 58, 233-311.

28. Kretzschmar, M. Eur. l. Phys. 1991, 12, 240-246.

29. Shockley, W. J. Appl. Phys. 1938, 9, 635.

30. Dunbar, R. C. Int. J. Mass Spectrom. Ion Proceedings 1984, 56, 1.

31. Nikolaev, E. N.; Gorshkov, M. V. Int. J. Mass Spectrom. Ion Proceedings 1985, 64, 115-125.

32. Brown, L. S.; Gabrielse, G. Phys. Rev. 1982, A25, 2423-2426.

33. Schweikhard, L. Int. I. Mass Spectrom. Ion Proceedings 1991. 107, 281-292.

34. Mitchell, D. W. Int. J. Mass Spectrom. Ion Proceedings 1991, 107, 417-434.

35. Comisarow, M. B. J. Chem. Phys. 1978, 69, 4097-4104.

36. Wang, M.; Marshall, A. G. Int. I. Mass Spectrom. Ion Proceedings 1990, 100, 323-346.

37. Gerz, C.; Wilsdorf, D.; Werth, G. Z. Phys. 1990, D17, 119-121.

38. Comisarow, M. B,; Marshall, A. G. Chem. Phys. Lett. 1974, 26, $489-490$.

39. Marshall, A. G.; Roe, D. C. J. Chem. Phys. 1980, 73, 1581-1590. 
40. Marshall, A. G.; Wang, T.-C. L.; Ricca, T. L. J. Am. Chem. Soc. 1985, 107, 7893-7897.

41. Marshall, A. G.; Wang, T.CC. L; Chen, L.; Ricca, T. L. In American Chemical Society; Buchanan, M. V., Ed.; American Chemical Society: Washington, DC, 1987; Symposium Series, Vol. 359; pp 21-33.

42. Mclver, R. T. Jr.; Hunter, R. L.; Baykut, G. Anal. Chem. 1989, 61, 489-491.

43. Rempel, D. L. Proceedings of the 31st ASMS Conference on Mass Spectrometry and Allied Topics; Boston, MA, 1983; pp 398-399.

44. Gorshkov, M. V.; Guan, S.; Marshall, A. G. Rapid Commun. Mass Spectrom. 1992, 6, 166-172.

45. Verdun, F. R.; Mullen, S. I..; Ricca, T. I.: Marshall, A. G. Presented at FACSS XIV; Detroit, MI. 1987; Abstract 41.

46. Fujiwara, M.; Katakura, H.; Inoue, M. Rapid Commun. Mass Spectrom. 1990, 4, 237-238.

47. Wachter, E. A.; Farrar, T. C.; Kontney, M. J. Int. J. Mass Spectrom. Ion Proceedings 1991, 103, 169-179.

48. Comisarow, M. B. In Ion Cyclotron Resonunce Spectrometry, Vol. II; Hartmann, H., Wanczek, K.-P., Eds.; Springer-Verlag: Berlin, 1982; pp 484-513.

49. Limbach, P. A.; Grosshans, P. B.; Marshall, A. G. Anal. Chem. $1993,65,135-140$

50. Grosshans, P. B.; Marshall, A. G. Int. I. Mass Spectrom. Ion Proceedings 1992, 115, 1-19.

51. Schweikhard, L.; Alber, G. M.; Marshall, A. G. J. Am. Sac. Mass Spectrom. 1993, 4, 177-181.

52. Schweikhard, L.; Alber, G. M.; Marshall, A. G. Physica Scripta 1992, 46, 598-602.

53. Guan, S.; Gorshkov, M. V.; Alber, G. M.; Marshall, A. G. Phys. Rev, A 1993

54. Dunbar, R. C.; Chen, J. H.; Hays, J. D. Int. J. Mass Spectrom. Ion Phys. 1984, 57, 39-56.

55. Williams, E. R.; Henry, K. D.; McLafferty, F. W. J. Am. Chem. Soc. 1990, 112, 6157-6162.

56. Beu, S. C.; Laude, D. A. Jr. Int. T. Mass Spectrom. Ion Proceedings 1991, 108, 255-268.

57. Rempel, D. L.; Huang, S. K.; Gross, M. L. Int. J. Mass Spectrom. Ion Proceedings 1986, 70, 163-184.
58. Huang, S. K.; Rempel, D. L.; Gross, M. L. Int. I. Mass Spectrom. Ion Proceedings 1986, 72, 15-31.

59. Allemann, M.; Kofel, P.; Kellerhals, H; Wanczek, K.-P. Int. J. Mass Spectrom. Ion Proceeindgs 1987, 75, 47-54.

60. van der Hart, W. J.; van de Guchte, W. J. Int. J. Mass Spectrom. Ion Proceedings 1988, 82, 17-31.

61. Wang, M.; Marshall, A. G. Anal. Chem. 1990, 62, 515-520.

62. Hanson, C. D.; Castro, M. E.; Kerley, E. L.; Russell, D. H. Anal. Chem. 1990, 62, 520-526.

63. van de Guchle, W. J., van der I Iart, W. J. Int. J. Mass Spectrom. Ion Proceedings 1990, 95, 317-326.

64. Hofstadler, S. A.; Laude, D. A., Jr. Int. J. Mass Spectrom. Ion Proceedings 1990, 101, 65-78.

65. Beu, S. C.; Laude, D. A., Jr. Anal. Chem. 1992, 64, 177-180.

66. Fujiwara, M.; Yamauchi, T.; Miura, K.; Inoue, M. J. Am. Soc. Mass Spectrom. 1992, 3,847-852.

67. Sommer, H.; Thomas, H. A.; Hipple, J. A. Phys. Rev. 1951, 82, $697-702$.

68. Caravatti, P.; Allemann, M. Org. Mass Spectrom. 1991, 26, 514-518.

69. Beu, S. C; Laude, D. A., Jr. Int. J. Mass Spectrom. Ion Proceedings 1992, 112, 215-230.

70. Fowles, G. R. Analytical Mechanics, 3rd ed.; Holt, Rinehart \& Winston: New York, 1977.

71. Stolzenberg, H.; Audi, G.; Becker, S.; Bollen, G.; Kern, F.; Kluge, H.-].; Moore, R. B.; Otto, T.; Savard, G.; Schweikhard, L. Phys. Rev. Lett. 1990, 65, 3104-3107.

72. Guan, S.; Marshall, A. G. J. Chem. Phys. 1993, 98, 4486-4493.

73. Wineland, D. J.; Dehmelt, H. G. Int. J. Mass Spectrom. Ion Phys. 1975, 16, 338.

74. Cornell, E. A.; Weisskoff, R. M.; Boyce, K. R.; Pritchard, D. E. Phys. Reo. 1990, A41, 312-315.

75. Van Dyck, R. S. Jr.; Schwinberg, P. B.; Dehmelt, H. G. In New Frontiers in High Energy Physics; Kursunoglu, B.; Pearlmutter, A.; Scott, L. F., Eds.; Plenum: New York, 1978; pp 159-181.

76. Kretzschmar, M. Physica Scripta 1992, 46, 544-554.

77. Kretzschmar, M. Physica Scripta 1992, 46, 555-559.

78. Mordehai, A. V.; Henion, J. D. Rapid. Commun. Mass Spectrom. 1992, 6, 345-348. 